\title{
Relations between frequency selectivity, temporal fine-structure processing, and speech reception in impaired hearing
}

Strelcyk, Olaf; Dau, Torsten

Published in:

Acoustical Society of America. Journal

Link to article, DOI:

10.1121/1.3097469

Publication date:

2009

Document Version

Publisher's PDF, also known as Version of record

Link back to DTU Orbit

Citation (APA):

Strelcyk, O., \& Dau, T. (2009). Relations between frequency selectivity, temporal fine-structure processing, and speech reception in impaired hearing. Acoustical Society of America. Journal, 125(5), 3328-3345.

https://doi.org/10.1121/1.3097469

\section{General rights}

Copyright and moral rights for the publications made accessible in the public portal are retained by the authors and/or other copyright owners and it is a condition of accessing publications that users recognise and abide by the legal requirements associated with these rights.

- Users may download and print one copy of any publication from the public portal for the purpose of private study or research.

- You may not further distribute the material or use it for any profit-making activity or commercial gain

- You may freely distribute the URL identifying the publication in the public portal 


\title{
Relations between frequency selectivity, temporal fine-structure processing, and speech reception in impaired hearing ${ }^{\text {a) }}$
}

\author{
Olaf Strelcyk and Torsten Dau \\ Centre for Applied Hearing Research, Department of Electrical Engineering, Technical University of \\ Denmark, Building 352, Ørsteds Plads, 2800 Kgs. Lyngby, Denmark
}

(Received 23 August 2008; revised 11 February 2009; accepted 12 February 2009)

\begin{abstract}
Frequency selectivity, temporal fine-structure (TFS) processing, and speech reception were assessed for six normal-hearing (NH) listeners, ten sensorineurally hearing-impaired (HI) listeners with similar high-frequency losses, and two listeners with an obscure dysfunction (OD). TFS processing was investigated at low frequencies in regions of normal hearing, through measurements of binaural masked detection, tone lateralization, and monaural frequency modulation (FM) detection. Lateralization and FM detection thresholds were measured in quiet and in background noise. Speech reception thresholds were obtained for full-spectrum and lowpass-filtered sentences with different interferers. Both the HI listeners and the OD listeners showed poorer performance than the NH listeners in terms of frequency selectivity, TFS processing, and speech reception. While a correlation was observed between the monaural and binaural TFS-processing deficits in the HI listeners, no relation was found between TFS processing and frequency selectivity. The effect of noise on TFS processing was not larger for the HI listeners than for the NH listeners. Finally, TFS-processing performance was correlated with speech reception in a two-talker background and lateralized noise, but not in amplitude-modulated noise. The results provide constraints for future models of impaired auditory signal processing. (C) 2009 Acoustical Society of America. [DOI: 10.1121/1.3097469]
\end{abstract}

PACS number(s): 43.71.Ky, 43.66.Sr, 43.66.Nm, 43.66.Pn [BCJM] Pages: 3328-3345

\section{INTRODUCTION}

Hearing-impaired (HI) people often experience great difficulty with speech communication when background noise is present. While audibility has been shown to be the main determinant of speech reception in quiet, it does not account to the same degree for speech reception in noise (e.g., Plomp, 1978; Dreschler and Plomp, 1985; Glasberg and Moore, 1989). Consequently, for many HI listeners, the problem persists even if reduced audibility has been compensated for by hearing aids. Other impairment factors besides reduced audibility must be involved.

Relations between frequency selectivity and speech reception, particularly in noise, have been reported previously (e.g., Festen and Plomp, 1983; Dreschler and Plomp, 1985; Horst, 1987; van Schijndel et al., 2001). Recently, also the processing of temporal fine-structure (TFS) information has received considerable attention with regard to speech reception (e.g., Tyler et al., 1983; Buss et al., 2004; Lorenzi et al., 2006; Hopkins et al., 2008). While envelope cues are sufficient to achieve good speech reception in quiet (e.g., Shannon et al., 1995), TFS cues may be required to ensure good speech reception in noise (e.g., Nie et al., 2005; Lorenzi and Moore, 2008). In particular, it has been suggested that deficits in TFS coding might account for the limited ability of HI listeners to take advantage of amplitude fluctuations in a noise background, i.e., to listen in the dips of a fluctuating interferer (e.g., Qin and Oxenham, 2003; Lorenzi et al., 2006; Gnansia et al., 2008). However, the large variability of

\footnotetext{
a) Portions of this work were presented at the 155th Meeting of the Acoustical Society of America, Paris, July 2008 (J. Acoust. Soc. Am. 123, 3712).
}

performance that is commonly observed across HI listeners makes it difficult to compare results across studies. Hence, only limited conclusions can be drawn about the relations between the different auditory functions, such as frequency selectivity and the processing of TFS. Also the relation between the deficits observed in monaural and binaural TFS processing remains unclear. Knowledge of these relations might shed light on the actual mechanisms and sites of the impairments.

Therefore, in the present study, individual performance on frequency selectivity, monaural and binaural TFS processing, and speech reception was measured using a common set of listeners. This is a similar concept to that used in the studies of Hall et al. (1984) and Gabriel et al. (1992), who examined binaural performance in individual HI listeners. Since the primary objective of the present study was to investigate impairment factors beyond audibility, ten $\mathrm{HI}$ listeners with similar high-frequency hearing losses were selected to provide a homogeneous group in terms of audibility. In this way, confounding effects of audibility were minimized and more direct conclusions could be drawn from a relatively small number of subjects about possible relations between the tested auditory functions. On the flip side, however, this group of HI listeners represents one homogeneous subset of the overall HI population and therefore one should act with caution in generalizing the results.

Besides the HI listeners, two further subjects were included in the present study. Despite normal audiograms, these subjects complained about difficulties with speech reception in noisy backgrounds. In literature, different terms have been used to refer to this phenomenon: auditory disabil- 
ity with normal hearing (King and Stephens, 1992), obscure auditory dysfunction (Saunders and Haggard, 1989), and King-Kopetzky syndrome (Hinchcliffe, 1992). For simplicity, in the present study, these subjects are referred to as having an obscure dysfunction (OD). In view of the heterogeneity of the clinical group of OD patients (e.g., Saunders and Haggard, 1989; Zhao and Stephens, 2000), these two listeners cannot constitute a representative sample and therefore should be regarded as cases. The comparison of performance between the two OD listeners and the HI listeners may provide valuable information on the nature of the underlying impairments in both groups.

Speech reception thresholds (SRTs) for full-spectrum and lowpass-filtered speech were measured in different diotic and dichotic interferers. The other psychoacoustic tests in this study were designed to examine basic auditory functions, mainly at a frequency of $750 \mathrm{~Hz}$. Low-frequency information has been shown to play a dominant role both for monaural abilities, such as the perception of pitch of complex tones (e.g., Terhardt, 1974; Moore et al., 1985), and for binaural abilities such as sound localization (e.g., Wightman and Kistler, 1992). Therefore, the frequency of $750 \mathrm{~Hz}$ was chosen to investigate the potential impact of a hearing impairment on auditory processing at low frequencies, even if a hearing loss in terms of elevated audiometric thresholds was present only at higher frequencies. As a basic auditory function, frequency selectivity was estimated via the notchednoise paradigm in simultaneous masking (e.g., Patterson and Nimmo-Smith, 1980).

Throughout the present study, the terms TFS information and TFS processing refer to the temporal fine structure at the output of the cochlear filters. This fine structure evokes phase-locked activity, i.e., synchronized timing of action potentials, in the subsequent stages of neural processing (see Ruggero, 1992, for a review). Apart from phase locking, TFS information may also be coded in terms of a conversion from frequency modulation to amplitude modulation (FM-to-AM) on the cochlear filter skirts, as has been suggested for the detection of high-rate FM (Zwicker, 1956; Moore, 2003). In the present study, however, the focus lies on TFS processing based on phase locking, rather than on the FM-to-AM conversion mechanism.

Evidence for TFS-processing deficits in HI listeners has been found in previous studies of monaural as well as binaural auditory functions. In terms of binaural processing, TFS deficits have been observed in the detection of interaural time or phase differences via lateralization (e.g., Hawkins and Wightman, 1980; Häusler et al., 1983; Smoski and Trahiotis, 1986; Gabriel et al., 1992; Koehnke et al., 1995; Lacher-Fougère and Demany, 2005). Also studies on binaural masked detection or masking level differences (MLDs) have reported deficits in HI listeners (e.g., Hall et al., 1984; Staffel et al., 1990; Gabriel et al., 1992). In both tasks, lateralization and binaural detection, the interaural phase or time differences in the stimuli can only be coded in terms of phase-locking-based TFS processing (see Stern and Trahiotis, 1995 and Colburn, 1996). Apart from these binaural measures of TFS processing, frequency discrimination of tones with frequencies of up to $4-5 \mathrm{kHz}$ is thought to be determined by a temporal mechanism based on phase locking (see Moore, 2003). Hence, deficits observed in the frequency discrimination of steady pure tones (e.g., Turner and Nelson, 1982; Tyler et al., 1983; Turner, 1987; Freyman and Nelson, 1991) and in the detection of low-rate FM (e.g., Zurek and Formby, 1981; Grant, 1987; Lacher-Fougère and Demany, 1998; Moore and Skrodzka, 2002; Buss et al., 2004) have been interpreted to indicate deficits in monaural TFS processing in HI listeners. This conclusion has been further supported by studies of frequency discrimination with harmonic complex tones (e.g., Horst, 1987; Moore et al., 2006; Hopkins and Moore, 2007). However, since none of the above mentioned studies has obtained both monaural and binaural measures of TFS processing, it remained unclear to what extent the deficits observed in the binaural tasks were due to monaural or independent binaural deficits.

Only a few studies have assessed the relation between TFS deficits and speech reception performance. Tyler et al. (1983), Glasberg and Moore (1989), Noordhoek et al. (2001), and Buss et al. (2004) found significant correlations between frequency discrimination performance and word recognition in speech-shaped noise ( $\mathrm{SSN}$ ) as well as quiet, while Horst (1987) did not find such correlations. Lorenzi et al. (2006) and Hopkins et al. (2008), using processed speech stimuli, presented evidence that HI listeners were less able to make use of the TFS information in speech than normal hearing $(\mathrm{NH})$ listeners. However, in these studies, the potential contribution of reduced frequency selectivity to the observed TFS deficits remained unclear. Reduced frequency selectivity might have affected the processing of TFS information in several ways (see also Moore, 2008). For wideband signals, the outputs of broadened auditory filters would exhibit a more complex TFS than the outputs of "normal" filters (Rosen, 1987). In addition, the signal-to-noise ratio (SNR) in the presence of a wideband interferer would be smaller in the case of broadened filters, providing a less favorable input to the subsequent processing stages. Finally, parts of the preserved TFS information in the speech stimuli of Lorenzi et al. (2006) might have been coded in terms of FM-to-AM conversion through cochlear filtering (e.g., Zeng et al., 2004; Gilbert and Lorenzi, 2006). In such a case, filter broadening would result in reduced AM depths at the filter output and a less distinct representation of frequency transitions (e.g., downward and upward glides) across adjacent filters. Hence, the observed deficits in the TFS processing of wideband stimuli could, in principle, have resulted from reduced frequency selectivity rather than from deficits in subsequent auditory processing stages.

Therefore, the present study investigated potential deficits in phase-locking-based TFS processing, where possible effects of frequency selectivity should play a minor role. Nevertheless, the relation between frequency selectivity and TFS processing was examined here since both might be affected by a common underlying impairment factor such as outer hair cell $(\mathrm{OHC})$ damage. The TFS processing was addressed binaurally through measurements of binaural masked detection and lateralization of pure tones with ongoing interaural phase differences (IPDs). As a complementary monaural measure, detection thresholds for low-rate frequency 
TABLE I. Audiometric information for the ten HI listeners and the two listeners with OD. The ears that were tested on monaural FM detection are marked by asterisks.

\begin{tabular}{|c|c|c|c|c|c|c|c|c|c|c|c|c|c|c|c|}
\hline \multirow[b]{2}{*}{ ID } & \multirow[b]{2}{*}{ Gender } & \multirow[b]{2}{*}{ Age } & \multirow[b]{2}{*}{ Ear } & \multicolumn{11}{|c|}{ Audiometric thresholds (dB HL) } & \multirow[b]{2}{*}{ Etiology } \\
\hline & & & & 125 & 250 & 500 & 750 & 1000 & 1500 & 2000 & 3000 & 4000 & 6000 & 8000 & \\
\hline \multirow[t]{2}{*}{$\mathrm{HI}_{1}$} & $\mathrm{~F}$ & 24 & $\mathrm{~L}^{*}$ & 5 & -5 & 0 & 5 & 15 & 25 & 35 & 60 & 60 & 55 & 60 & \\
\hline & & & $\mathrm{R}$ & 0 & -5 & 0 & 5 & 15 & 25 & 30 & 55 & 55 & 65 & 70 & Hypoxia at birth \\
\hline \multirow[t]{2}{*}{$\mathrm{HI}_{2}$} & M & 53 & $\mathrm{~L}$ & 5 & 0 & 5 & 10 & 5 & 15 & 30 & 45 & 40 & 50 & 55 & \\
\hline & & & $\mathrm{R}^{*}$ & 5 & 5 & 0 & 10 & 5 & 20 & 30 & 40 & 45 & 55 & 60 & Unknown \\
\hline \multirow[t]{2}{*}{$\mathrm{HI}_{3}$} & M & 55 & $\mathrm{~L}^{*}$ & 0 & 5 & 10 & 15 & 15 & 10 & 55 & 70 & 55 & 60 & 55 & \\
\hline & & & $\mathrm{R}$ & 0 & 5 & 15 & 15 & 20 & 10 & $30^{\mathrm{a}}$ & 60 & 65 & 55 & 60 & Noise induced \\
\hline \multirow[t]{2}{*}{$\mathrm{HI}_{4}$} & M & 56 & $\mathrm{~L}^{*}$ & 5 & 5 & 5 & 5 & 5 & 25 & 30 & 45 & 45 & 55 & 70 & \\
\hline & & & $\mathrm{R}$ & -5 & 0 & -5 & 5 & 5 & 15 & 25 & 50 & 50 & 55 & 65 & Hereditary \\
\hline \multirow[t]{2}{*}{$\mathrm{HI}_{5}$} & M & 60 & $\mathrm{~L}^{*}$ & 10 & 5 & 10 & 10 & 5 & 35 & 60 & 60 & 60 & 55 & 60 & \\
\hline & & & $\mathrm{R}$ & 0 & 0 & 5 & 10 & 5 & 40 & 50 & 65 & 60 & 60 & 60 & Noise induced \\
\hline \multirow[t]{2}{*}{$\mathrm{HI}_{6}$} & $\mathrm{M}$ & 67 & $\mathrm{~L}^{*}$ & 0 & 5 & 5 & 10 & 10 & 15 & 30 & 45 & 50 & 50 & 65 & \\
\hline & & & $\mathrm{R}$ & 0 & 5 & 5 & 10 & 5 & 15 & 20 & 45 & 60 & $65^{\mathrm{a}}$ & 65 & Unknown \\
\hline \multirow[t]{2}{*}{$\mathrm{HI}_{7}$} & M & 70 & $\mathrm{~L}$ & 5 & 5 & 0 & 10 & 15 & 10 & 20 & 50 & 65 & 60 & 60 & \\
\hline & & & $\mathrm{R}^{*}$ & 5 & 10 & 0 & 10 & 15 & 15 & 15 & 55 & 55 & 60 & 65 & Noise induced \\
\hline \multirow[t]{2}{*}{$\mathrm{HI}_{8}$} & $\mathrm{~F}$ & 70 & $\mathrm{~L}^{*}$ & 5 & 5 & 15 & 20 & 20 & 30 & 45 & 60 & 65 & 60 & 60 & \\
\hline & & & $\mathrm{R}$ & 5 & 5 & 10 & 15 & 20 & 35 & 45 & 60 & 60 & 60 & 55 & Unknown \\
\hline \multirow[t]{2}{*}{$\mathrm{HI}_{9}$} & M & 74 & $\mathrm{~L}$ & 20 & 10 & 5 & 10 & 20 & 40 & 35 & 60 & 60 & 60 & 70 & \\
\hline & & & $\mathrm{R}^{*}$ & 20 & 15 & 5 & 10 & 10 & 50 & $60^{\mathrm{a}}$ & 60 & 60 & 55 & $55^{\mathrm{a}}$ & Noise induced \\
\hline \multirow[t]{2}{*}{$\mathrm{HI}_{10}$} & $\mathrm{~F}$ & 74 & $\mathrm{~L}^{*}$ & 0 & 10 & 10 & 15 & 20 & 45 & 55 & 65 & 65 & 60 & 70 & \\
\hline & & & $\mathrm{R}$ & 5 & 5 & 10 & 15 & 15 & 35 & 55 & 55 & 60 & 60 & 60 & Noise induced \\
\hline \multirow[t]{2}{*}{$\mathrm{OD}_{1}$} & $\mathrm{~F}$ & 26 & $\mathrm{~L}$ & -5 & -5 & 0 & 0 & 0 & 0 & 0 & 0 & 0 & 10 & 5 & \\
\hline & & & $\mathrm{R}^{*}$ & -5 & -5 & 0 & 0 & 0 & 0 & 0 & 5 & 0 & 5 & 5 & None \\
\hline \multirow[t]{2}{*}{$\mathrm{OD}_{2}$} & $\mathrm{~F}$ & 46 & $\mathrm{~L}$ & 0 & 5 & 5 & 5 & 10 & 5 & 5 & 5 & 0 & 10 & 5 & \\
\hline & & & $\mathrm{R}^{*}$ & 0 & 0 & 0 & 5 & 10 & 5 & 10 & 0 & 10 & 10 & 5 & None \\
\hline
\end{tabular}

${ }^{\mathrm{a}}$ Thresholds differ by more than $10 \mathrm{~dB}$ between the ears.

modulation (FMDTs) were obtained. The IPD thresholds and FMDTs were measured in quiet as well as in continuous noise backgrounds in order to test the robustness of the TFS processing to interfering noise. Physiological animal studies (e.g., Rhode et al., 1978; Abbas, 1981; Costalupes, 1985) have shown that phase locking to tones in the presence of background noise is generally preserved at SNRs near behavioral detection thresholds but ceases at sufficiently low SNRs. However, as no comparable studies exist in impaired hearing, it cannot be excluded that hearing impairment might potentiate the susceptibility of phase locking to noise disturbance.

\section{METHODS}

\section{A. Listeners}

The six NH listeners (three females and three males) were aged between 21 and 55 years (median: 28) and had audiometric thresholds better than $20 \mathrm{~dB}$ hearing level
(HL; ISO 389-8, 2004) at all octave frequencies from 125 to $8000 \mathrm{~Hz}$ and 750 to $6000 \mathrm{~Hz}$. The ten HI listeners (three females and seven males) were aged between 24 and 74 years (median: 63). Detailed audiometric information is given in Table I. Throughout the study, the HI subjects are sorted by age and the notation " $\mathrm{HI}_{n}$ " is used to refer to the individual subject with index $n$. The audiograms were "normal" up to $1 \mathrm{kHz}$ (thresholds $\leq 20 \mathrm{~dB} \mathrm{HL}$ ) and sloping at higher frequencies to values of up to $70 \mathrm{~dB}$ HL. All listeners had bilaterally symmetric audiograms (within $10 \mathrm{~dB}$, exceptions stated in Table I), to avoid the issue of level balancing in binaural testing, as discussed in Durlach et al., 1981. The sensorineural origin of the hearing losses was established by means of bone-conduction measurements, tympanometry, and otoscopy. The etiologies stated in Table I were based on the subjects' reports. They ranged from hypoxia at birth (oxygen deficiency) and hereditary losses to noise-induced losses, either sudden or due to sustained exposure to intense sounds. The remaining two subjects had OD: Despite audio- 
metric thresholds better than $15 \mathrm{~dB} \mathrm{HL}$ at all test frequencies (see Table I), they approached the research center, complaining about difficulties with understanding speech in noisy backgrounds. Their middle-ear status was normal and they did not report any history of otitis media or excessive noise exposure. Auditory brainstem responses were measured for these two subjects and the $\mathrm{HI}$ subject $\mathrm{HI}_{10}$ ( since $\mathrm{HI}_{10}$ showed diverging results in the lateralization task). As the responses were normal, there was no indication of eighthnerve tumors, brainstem lesions, or auditory neuropathy. Additionally, all listeners were screened on a binaural pitch task, testing the ability to hear a Huggins' pitch $C$-scale (Santurette and Dau, 2007). Santurette and Dau (2007) suggested that the absence of a binaural pitch percept might indicate the presence of a severe central auditory deficiency. Since all listeners in the present study perceived the pitch there was no indication of such a deficiency.

Each subject completed all tests, with the exception of one NH listener, for whom SRTs were not measured. The average testing time was $24 \mathrm{~h}$ per listener. All experiments were approved by the Ethics Committee of Copenhagen County.

\section{B. Apparatus}

All stimuli were generated in MATLAB $®$ and converted to analog signals using a 24-bit digital-to-analog converter (RME DIGI96/8). The sampling rate was $44.1 \mathrm{kHz}$ for the speech reception measurement, $48 \mathrm{kHz}$ for the masking and FM experiments, and $96 \mathrm{kHz}$ for the lateralization task. The stimuli were presented in a double-walled sound-attenuating booth via Sennheiser HD580 headphones. Calibrations were done using a B\&K 4153 artificial ear and, prior to playing, 128-tap linear-phase FIR equalization filters were applied to all broadband stimuli, rendering the headphone frequency response flat.

\section{Statistical analyses}

To accommodate the repeated-measures design, the statistical analyses were carried out using linear mixed-effects models (Laird and Ware, 1982; Pinheiro and Bates, 2000), as implemented in S-PLUS®. The between-subject variability that was not explained in terms of the fixed effect subject group (or interactions of other fixed effects such as stimulus condition with subject group) was accounted for in terms of subject-specific random effects. In addition to analyses of variance (ANOVAs) and multiple comparisons of the fixed effects (with simultaneous $95 \%$ confidence intervals, either based on the Dunnett method or Monte Carlo simulations), the estimated random effects were extracted. They served as ranks for the individual listeners' performance on a given test, for example, binaural masked detection or lateralization. In the following, the abbreviations SD and CI will be used for standard deviation and confidence interval, respectively.

\section{SPEECH RECEPTION}

\section{A. Method}

SRTs were measured for Danish closed-set Hagerman sentences (Dantale II, Wagener et al., 2003) in the presence of different interferers: a stationary SSN with the long-term spectrum of the Dantale II sentences, a sinusoidally and a randomly amplitude-modulated noise (SAM and RAM), a multitalker and a reversed two-talker background (MULTITALK and TWOTALK), and a dichotic, lateralized speechshaped noise (LATSSN). Specifically, the SAM noise was fully sinusoidally amplitude-modulated SSN, with a modulation rate of $8 \mathrm{~Hz}$ (cf. Füllgrabe et al., 2006). The RAM noise was randomly amplitude-modulated SSN, with the Hilbert envelope of a $20-\mathrm{Hz}$-wide noise used as modulator. The MULTITALK noise was a reversed 20-talker babble (supplied as track 3 on compact disk CD101R3 "Auditory Tests Revised" by AUDiTEC of St. Louis). The TWOTALK noise consisted of running female and male speech, with silent gaps longer than $250 \mathrm{~ms}$ removed, mixed at equal level, and time-reversed (speech supplied as tracks 8 and 9 on compact disk CD B\&O 101 "Music for Archimedes" by Bang \& Olufsen). The LATSSN (noise) was SSN, which was lateralized to one side by means of a constant interaural time difference of $740 \mu \mathrm{s}$. For a given run, either the left or the right ear was leading, but the SRT was averaged across runs with lateralization to the left and right. In addition to these conditions with full-spectrum speech, two conditions with filtered speech were used, $\mathrm{SSN}_{\text {filt }}$ and $\mathrm{SAM}_{\text {filt }}$, in which both target speech and interferer were lowpass filtered at $1 \mathrm{kHz}$ (1024-tap FIR lowpass filter designed using the ParksMcClellan algorithm in MATLAB () . This was done to test the processing of speech information in the regions of normal hearing (as all listeners had normal audiometric thresholds up to $1 \mathrm{kHz}$ ). The SRTs in all the aforementioned conditions were measured binaurally with the target speech and interferer presented diotically, with the exception of the LATSSN condition, where the interferer was presented dichotically. In addition, SRTs in the SSN and SAM conditions were measured monaurally, for comparison with the other monaural tests of frequency selectivity and FM detection.

The SRT was defined as the SNR leading to 50\% correct identification of the individual words in the Dantale II sentences. The interferer level was kept constant at $65 \mathrm{~dB}$ sound-pressure-level (SPL) while the sentence level was varied adaptively. In each condition, the listeners were trained on a single run of 20 sentences. Subsequently, the SRT was estimated as the average over two to three runs, depending on the condition. A monotonic improvement of threshold in a sequence of three runs was interpreted as a training effect. When such an effect occurred, further runs were taken until stable performance was reached, and the first runs were discarded. This procedure for dealing with training effects was applied to all the other tests in this study.

\section{B. Results and discussion}

Figure 1 shows the binaural SRTs for the NH (circles), the OD (bold numbers), and the HI listeners (plain numbers). The horizontal black bars denote the mean SRTs for the NH 


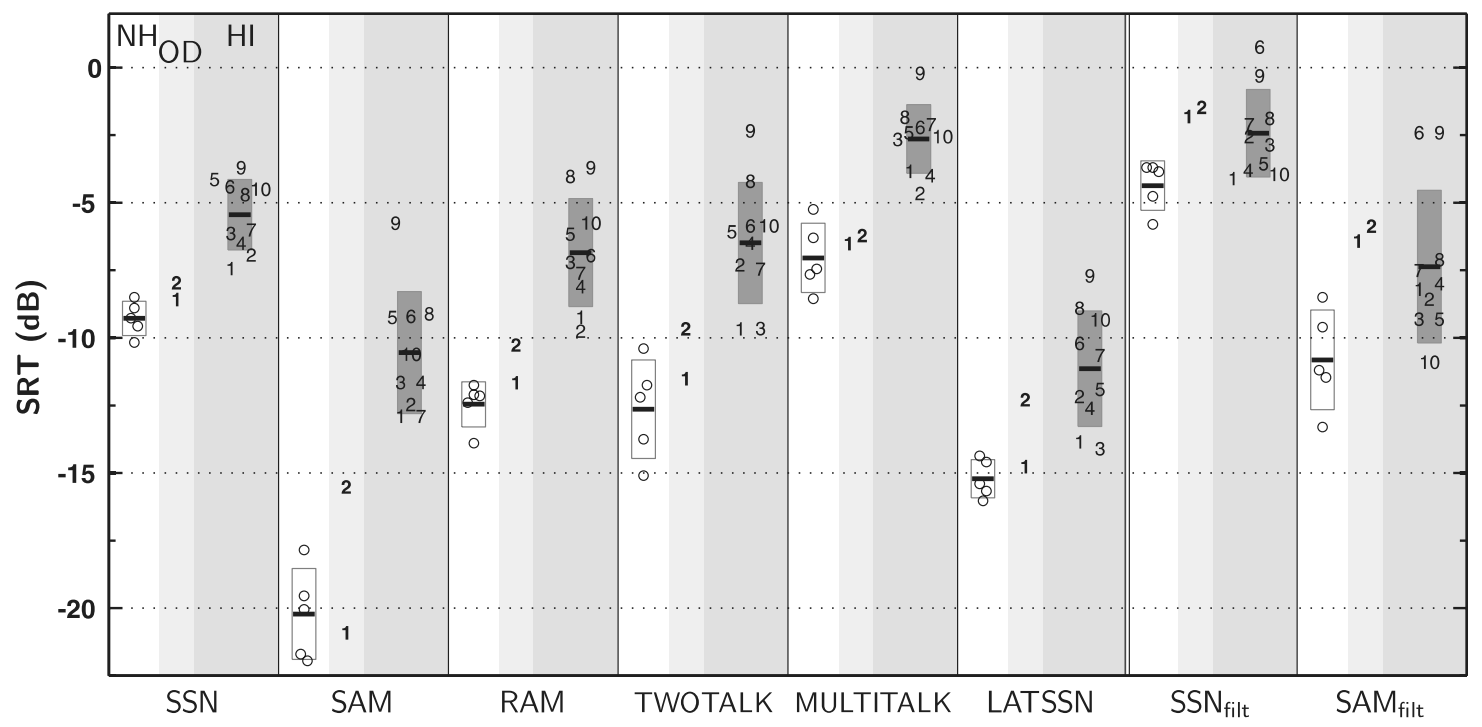

FIG. 1. SRTs for the NH listeners (circles), the two listeners with OD (bold numbers), and the HI listeners (plain numbers). The different conditions are indicated at the bottom of each panel. The horizontal black bars show the mean SRTs for the NH and HI listeners and the corresponding boxes represent $\pm 1 \mathrm{SD}$.

and HI listeners and the corresponding boxes represent \pm 1 SD. Considering the first six conditions with full-spectrum speech, all listeners showed the lowest SRTs with the SAM and LATSSN interferers, while the highest SRTs were obtained with the MULTITALK interferer. The SRTs for the RAM and TWOTALK conditions lay slightly below those for the stationary SSN interferer. Performance in the conditions with lowpass-filtered speech $\left(\mathrm{SSN}_{\text {filt }}\right.$ and $\left.\mathrm{SAM}_{\text {filt }}\right)$ was generally poorer than performance in the corresponding conditions with full-spectrum speech. An ANOVA was performed on the SRTs of the NH and HI listeners. The SRTs were found to be significantly higher for the HI listeners than for the NH listeners $[F(1,13)=36.1, p<0.0001]$. The SRTs differed significantly across conditions $[F(7,91)=238.7$, $p<0.0001]$ and the interaction between listener group and condition was significant $[F(7,91)=20.5, p<0.0001]$. Multiple comparisons revealed that the HI listeners performed more poorly than the NH listeners for all full-spectrum conditions $[p<0.001]$. For the two conditions with lowpassfiltered speech, the HI listeners' deficits were less pronounced. The deficit was significant for the $\mathrm{SAM}_{\text {filt }}$ condition $[p<0.01]$, but not for the $\operatorname{SSN}_{\text {filt }}$ condition $[p>0.05]$. Within the group of HI listeners, no significant correlation was observed between the SRTs for the filtered speech and the full-spectrum speech $[p>0.05]$. Hence, they did not seem to make equally good use of the low-frequency and high-frequency information in the speech stimuli. For example, listeners $\mathrm{HI}_{5}$ and $\mathrm{HI}_{10}$ performed relatively well in the filtered-speech task, but poorly in the full-spectrum speech task.

Previously, Horwitz et al. (2002) measured speech reception performance of $\mathrm{HI}$ listeners in regions of normal hearing, using lowpass-filtered speech in a SSN masker. In contrast to the present results $\left(\mathrm{SSN}_{\text {filt }}\right.$ condition), they found significantly poorer performance for their HI than for their NH listeners. However, their speech stimuli were presented at a level of $77 \mathrm{~dB}$ SPL, where a substantial spread of exci- tation on the basilar membrane would be expected, particularly toward places corresponding to higher characteristic frequencies. Consequently, they interpreted their finding in terms of a reduced ability of their HI listeners to encode the information at places with high characteristic frequencies, where a hearing loss was present.

In addition to the SRTs, speech masking release was considered, i.e., the gain in terms of SRT for the SAM, RAM, TWOTALK, and LATSSN conditions when compared with the SRT for the stationary, diotic SSN condition. The group masking release values can be extracted from Fig. 1 as the differences in SRT between the corresponding conditions. The masking release values were significantly smaller for the HI listeners than for the $\mathrm{NH}$ listeners $[F(1,13)$ $=21.8, p=0.0004]$. While the SAM masking release values for the full-spectrum speech differed strongly between the $\mathrm{NH}$ and $\mathrm{HI}$ listeners [by $5.8 \mathrm{~dB}, p<0.001$ ], the difference for the filtered speech just reached significance $[1.5 \mathrm{~dB}$, $p=0.05]$. The finding of less pronounced deficits with lowpass-filtered speech may, at least partly, be attributed to the fact that the HI listeners had normal low-frequency hearing thresholds and that the full-spectrum speech stimuli were not amplified to fully restore audibility at high frequencies. It is interesting that the HI listeners did not benefit from highfrequency information in terms of the SAM masking release: While the NH listeners showed a significantly larger masking release with full-spectrum speech (SAM-SSN) than with filtered speech $\left(\mathrm{SAM}_{\text {filt }}-\mathrm{SSN}_{\text {filt }}\right)$ [difference in $\mathrm{dB}: 4.5$ (CI 3.3, 5.7)], the difference was not significant for the HI listeners $[0.2(\mathrm{CI}-0.9,1.2) \mathrm{dB}]$.

As mentioned above, higher SRTs were observed in the MULTITALK masker than in the SSN masker, independent of listener group. Hence, in addition to the energetic masking present for the latter, another detrimental masking effect must have limited speech intelligibility in the case of the MULTITALK background. This could, for example, have been the complex harmonic structure of the background 
babble, which interfered with the use of spectro-temporal cues in the target speech, such as formant transitions.

As can be seen in Fig. 1, the OD listeners showed rather small deficits in the reception of full-spectrum speech. Consistent with previous reports in literature (e.g., Middelweerd et al., 1990; Saunders and Haggard, 1992), they often performed at the lower limit of the $\mathrm{NH}$ group. Subject $\mathrm{OD}_{1}$ showed elevated SRTs only in the two filtered-speech conditions. Subject $\mathrm{OD}_{2}$ showed poorer performance than the $\mathrm{NH}$ listeners in all conditions except MULTITALK. Particularly in the SAM, LATSSN, and both filtered-speech conditions, her SRTs were increased relative to those for the NH group. Hence, for these two listeners, a deficit with speech reception was most apparent for the lowpass-filtered speech. This deficit might reflect a general difficulty understanding speech that is less redundant than full-spectrum speech. However, it could also reflect a specific problem with the processing of low-frequency information.

The monaural SRTs, which were measured only in the SSN and SAM conditions, closely followed the corresponding binaural results described above (monaural SRTs were, on average, $1.5 \mathrm{~dB}$ higher than binaural SRTs). The mean monaural SSN SRT of -8.7 (SD 0.9) dB for the NH listeners was consistent with the SRT of -8.4 (SD 1.0) dB reported by Wagener et al. (2003). Since the monaural results do not provide any further insights they are not presented in detail.

\section{FREQUENCY SELECTIVITY}

\section{A. Method}

Auditory-filter shapes at $750 \mathrm{~Hz}$ were determined separately for each ear using a notched-noise paradigm (cf. Patterson and Nimmo-Smith, 1980). Rosen et al. (1998) presented evidence that auditory-filter shapes are output driven. Under the assumption of the power-spectrum model (cf. Patterson and Moore, 1986) that a constant SNR at the output of the auditory filter is required for detection, this is equivalent to saying that the filter shape is determined by the level of the target signal rather than the noise masker. Therefore, here, in order to obtain a faithful filter estimate, the signal level was kept constant while the masker level was varied adaptively. The $750-\mathrm{Hz}$ target tones of $440-\mathrm{ms}$ duration were presented at a fixed level of $50 \mathrm{~dB}$ SPL and were temporally centered in the 550-ms noise maskers. Maskers and tones were gated with $50-\mathrm{ms}$ raised-cosine ramps. The noise was generated in the spectral domain as fixedamplitude random-phase noise (this holds also for the noises in all remaining tests). Five symmetric $\left(\delta f / f_{0}: 0.0,0.1,0.2\right.$, 0.3 , and 0.4$)$ and two asymmetric notch conditions $\left(\delta f / f_{0}: 0.2 \mid 0.4\right.$ and $\left.0.4 \mid 0.2\right)$ were used, where $\delta f$ denotes the spacing between the inner noise edges and the signal frequency $f_{0}$. The outside edges of the noise maskers were fixed at $\pm 0.8 f_{0}$.

A three-interval, three-alternative, forced-choice (3I3AFC) weighted up-down method (Kaernbach, 1991) was applied to track the $75 \%$ correct point on the psychometric function. A run was terminated after 14 reversals. The threshold was defined as the arithmetic mean of all masker levels following the fourth reversal. Following a training run for (a)

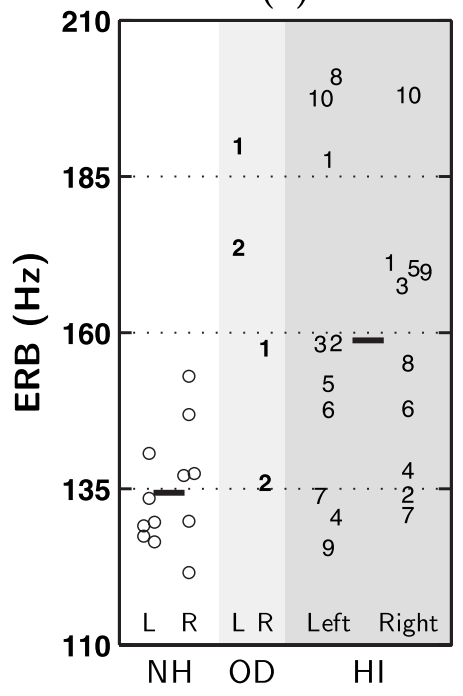

(b)

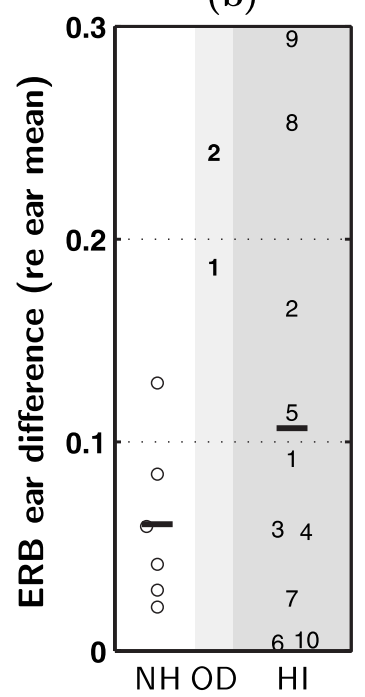

FIG. 2. (a) ERB of the $\operatorname{roex}(p, r)$ filter estimates at $750 \mathrm{~Hz}$ for the $\mathrm{NH}$ listeners (circles), the two listeners with OD (bold numbers), and the HI listeners (plain numbers). For each group, the left and right symbols or numbers correspond to the left and right ears, respectively. The horizontal black bars denote group means. (b) Absolute value of the ERB differences between the ears, divided by the mean ERB for the two ears.

each notch condition, the threshold was estimated as the average over three runs. If the SD of these three runs exceeded $1 \mathrm{~dB}$, one or two additional runs were taken and the average of all was used.

A nonlinear minimization routine was implemented in $M A T L A B \circledR$ to find the best-fitting rounded-exponential filter in the least-squares sense, assuming that the signal was detected using the filter with the maximum SNR at its output. Middle-ear filtering was taken into account, using the middle-ear transfer function supplied by Moore et al. (1997). However, the results presented in the following do not depend on this choice. Furthermore, besides the equivalent rectangular bandwidth (ERB) as a measure of filter tuning, also the $3-\mathrm{dB}$ and $10-\mathrm{dB}$ bandwidths were considered. However, because they yielded essentially identical results, for ease of comparison only the ERB results will be discussed further.

\section{B. Results and discussion}

The roex $(p, r)$ filter model (Patterson et al., 1982) provided a good description of the individual notched-noise threshold data, with a rms fitting error of 0.64 (SD 0.25) dB, averaged across all subjects. ${ }^{1}$ Figure 2(a) shows the estimated ERBs for the $\mathrm{NH}$ and $\mathrm{HI}$ listeners as well as the two OD listeners. The HI listeners showed, on average, significantly higher bandwidths than the $\mathrm{NH}$ listeners $[F(1,14)$ $=13.5, p=0.003]$, by a factor of 1.2. However, the results varied considerably across the HI listeners, with four of them showing bandwidths in both ears within the range of the $\mathrm{NH}$ listeners. In addition to the ERB, significantly shallower lower and upper filter skirts were observed for the HI listeners than for the $\mathrm{NH}$ listeners [lower skirt: $F(1,14)$ $=10.9, p=0.005$; upper skirt: $F(1,14)=5.6, p=0.03]$. 


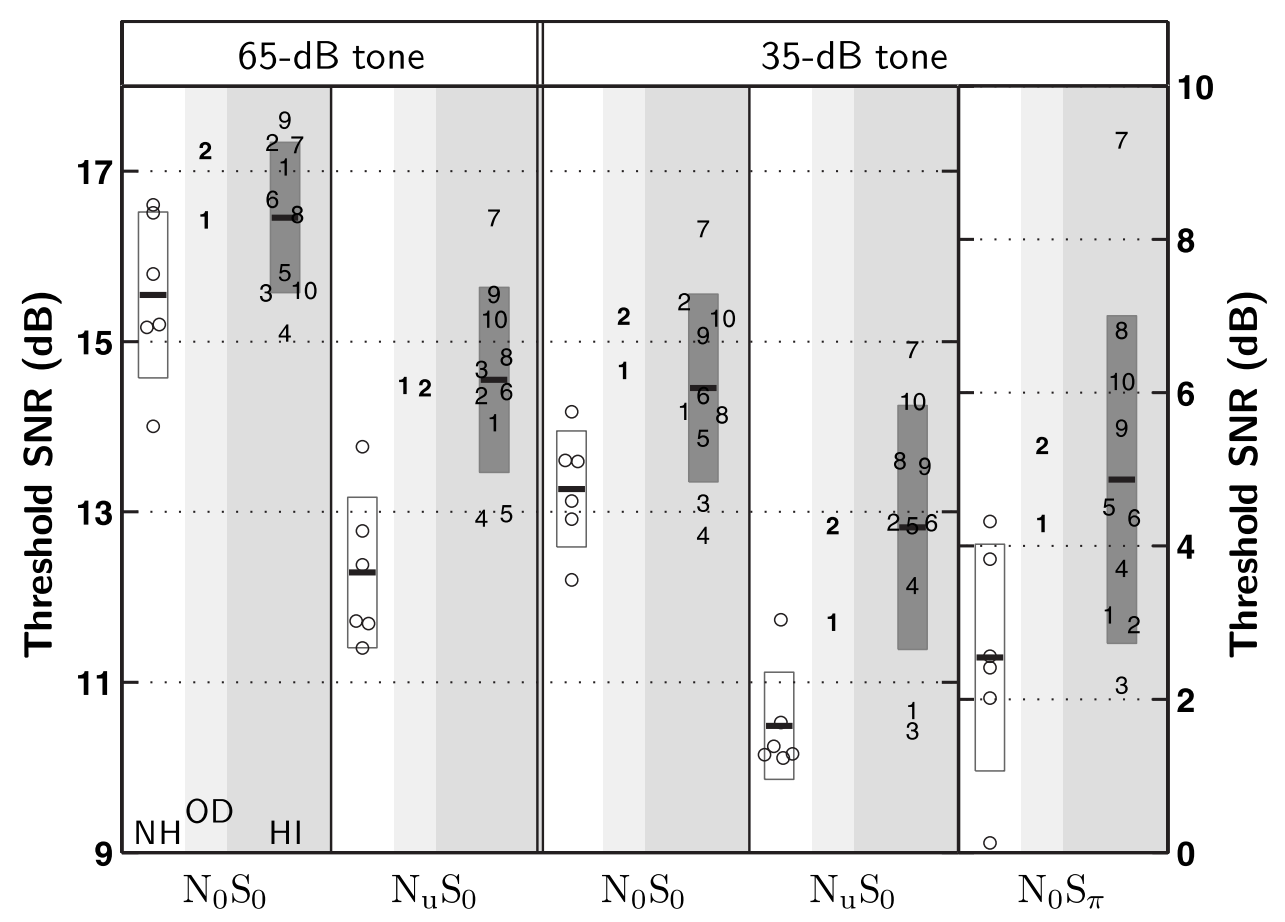

FIG. 3. Binaural masked thresholds, i.e., tone level re masker spectrum level at detection threshold, for the $\mathrm{NH}$ listeners (circles), the two listeners with OD (bold numbers), and the HI listeners (plain numbers), obtained in three different masking conditions $\left(N_{0} S_{0}, N_{u} S_{0}\right.$, and $\left.N_{0} S_{\pi}\right)$ and at two different tone levels ( 65 and $35 \mathrm{~dB}$ SPL). Note the different offset of the ordinate for the $N_{0} S_{\pi}$ condition. Otherwise as Fig. 1.

As can be seen in Fig. 2(a), abnormal filter bandwidths were also found for the two OD listeners. While $\mathrm{OD}_{1}$ showed significantly elevated bandwidths (compared to the $\mathrm{NH}$ group) in both ears, $\mathrm{OD}_{2}$ showed an increased bandwidth only in the left ear. The difference between the ERB of the left and right ears (divided by the mean ERB of the two ears) is depicted in Fig. 2(b). While this interaural bandwidth asymmetry did not differ significantly between the $\mathrm{NH}$ and HI listeners, both OD subjects showed larger differences between the ears than the $\mathrm{NH}$ listeners and most of the $\mathrm{HI}$ listeners. Decreased frequency selectivity, as found here, has been reported previously in the OD literature (e.g., Narula and Mason, 1988; Saunders and Haggard, 1992). It is also consistent with the finding of reduced distortion-product otoacoustic emission amplitudes (Zhao and Stephens, 2006), if these are taken as an indication of $\mathrm{OHC}$ integrity.

The mean ERB of 134 (SD 9) $\mathrm{Hz}$ for the NH listeners is larger than the value of $106 \mathrm{~Hz}$ predicted by the ERB function given in Glasberg and Moore, 1990. However, that function was designed to predict tuning in the presence of a masker with a constant spectrum level of about $35 \mathrm{~dB}$ SPL. It is known that the auditory-filter bandwidth increases with increasing (output) level (e.g., Rosen et al., 1998). Hence, the larger bandwidths found here may be attributed to the higher masker levels applied (the average spectrum level here was $48 \mathrm{~dB}$ SPL). In fact, they are in good agreement with the bandwidths reported by Moore et al. (1990) who measured at comparable masker levels.

\section{BINAURAL MASKED DETECTION}

\section{A. Method}

The binaural masked thresholds for $750-\mathrm{Hz}$ tones at fixed levels of 65 and $35 \mathrm{~dB}$ SPL were measured in bandlimited noise $(50-1500 \mathrm{~Hz})$. Three different masking conditions were tested: a diotic tone presented in a diotic noise
$\left(N_{0} S_{0}\right)$, a diotic tone presented in an uncorrelated noise $\left(N_{u} S_{0}\right)$, and a tone with an interaural phase shift of $180^{\circ}$ presented in a diotic noise $\left(N_{0} S_{\pi}\right)$. The first two conditions were measured using both tone levels whereas the last condition was measured only for the lower tone level. The tones of 500-ms duration were temporally centered in the 700-ms noise maskers. Maskers and tones were gated with raisedcosine ramps of 100-ms and 200-ms durations, respectively.

The same 3I-3AFC method as for the frequency selectivity measurement (including threshold estimation) was used. Also here, the signal level was kept constant while the masker level was varied adaptively. The final standard error of the masked threshold estimate, averaged across all listeners and conditions, was $0.4 \mathrm{~dB}$.

\section{B. Results and discussion}

The masked thresholds for the NH, the OD, and the HI listeners are shown in Fig. 3, with SNRs given relative to the masker spectrum level. For all listeners, the thresholds were lower in the dichotic $N_{u} S_{0}$ and $N_{0} S_{\pi}$ conditions than in the corresponding diotic $N_{0} S_{0}$ conditions. These MLDs reflected a release from masking in the dichotic configurations and will be discussed further below. An ANOVA revealed that the masked thresholds were significantly higher for the HI than for the $\mathrm{NH}$ listeners $[F(1,14)=14.7, p=0.002]$. Furthermore, the masked thresholds differed significantly between the different binaural conditions $[F(2,59)=536.9$, $p<0.0001]$ and also the interaction between listener group and masking condition was significant $[F(2,59)=4.2$, $p=0.02]$. While there was no significant difference between the $\mathrm{NH}$ and $\mathrm{HI}$ listeners for the diotic $N_{0} S_{0}$ condition [group difference: $1.1(\mathrm{CI}-0.3,2.4) \mathrm{dB}$, thresholds for the dichotic conditions differed significantly $\left[N_{u} S_{0}\right.$ group difference: 2.3 (CI 1.0, 3.6) dB; $N_{0} S_{\pi}$ group difference: 2.3 (CI 0.8 , 3.9) $\mathrm{dB}$ ]. Furthermore, within the group of HI listeners, a 
significant correlation between the $N_{u} S_{0}$ and $N_{0} S_{\pi}$ thresholds was observed $[r=0.87, p=0.001]$. Together, this suggests a deficit with TFS processing at threshold, which impaired $N_{u} S_{0}$ and $N_{0} S_{\pi}$ detections in similar ways.

Significantly larger SNRs were required for the detection of the 65-dB tones than for the 35-dB tones [effect of level on SNR: 1.9 (CI 1.5, 2.4) dB]. This is consistent with the notion of decreasing sharpness of the auditory filters with increasing tone level, if detector efficiency is assumed to be invariant (as found by Rosen et al., 1998). However, as can also be seen in Fig. 3 (mean results), the effect of tone level did not differ significantly across masking condition or listener group. The latter is in agreement with Baker and Rosen (2002), who found a differential effect of tone level on the ERBs of their $\mathrm{NH}$ and $\mathrm{HI}$ listeners only for levels above $70 \mathrm{~dB}$ SPL.

The following MLDs were observed for the NH listeners: $\quad N_{0} S_{0}-N_{u} S_{0} 3.0$ (SD 0.7) dB and $N_{0} S_{0}-N_{0} S_{\pi} 10.7$ (SD 1.3) dB. Since tone level had no significant effect, here, the $N_{0} S_{0}-N_{u} S_{0}$ MLD was averaged across the two tone levels. The HI listeners showed significantly smaller $N_{0} S_{0}-N_{u} S_{0}$ MLDs than the NH listeners [reduced by 1.3 (CI $0.1,2.4) \mathrm{dB}$ ]. However, no significant difference was found for the $N_{0} S_{0}-N_{0} S_{\pi}$ MLD [reduced by 1.1 (CI -0.2 , 2.5) dB]. Hence, the deficits in terms of the MLDs were less significant than the deficits in terms of the masked thresholds. This was due to the fact that the HI listeners exhibited not only significantly increased dichotic thresholds, but also slightly increased diotic thresholds, as previously reported by Staffel et al. (1990) and Gabriel et al. (1992).

Figure 3 also shows the masked threshold results for the two OD listeners. While subject $\mathrm{OD}_{2}$ performed clearly more poorly than the $\mathrm{NH}$ listeners, subject $\mathrm{OD}_{1}$ showed performance at the "lower edge" of that for the NH group. However, this applied to both the diotic and the dichotic masking conditions, as reported previously by Saunders and Haggard (1992). Therefore, in terms of their MLDs, no deficits were found for the OD listeners.

\section{LATERALIZATION}

\section{A. Method}

Lateralization thresholds were measured for $750-\mathrm{Hz}$ tones of 500-ms duration, at fixed levels of 70 and $35 \mathrm{~dB}$ SPL. The tones were gated synchronously and were lateralized by introducing a carrier-phase delay to one of the ears, giving rise to an IPD. For NH listeners, interaural carrier delays have been shown to dominate interaural gating delays for frequencies below about $1.5 \mathrm{kHz}$ (see Zurek, 1993). To further weaken potential gating cues to lateralization, long onset/offset ramps of $200 \mathrm{~ms}$ each were used. Pilot measurements confirmed that the lateralization was solely based on TFS cues, since no significant difference was found between the lateralization thresholds for tones with a waveform delay and tones with a carrier delay only. At each tone level, in addition to the lateralization threshold in quiet, three conditions with different bandlimited noise interferers $(50-1500 \mathrm{~Hz})$ were measured: diotic noise at a low (dioticLo) and a high sound level (dioticHi), and dichotic noise at an intermediate level (dichotic). The noise level in each condition was chosen relative to the individual's masked threshold $\left(N_{0} S_{0}\right.$ or $\left.N_{u} S_{0}\right)$ to make sure that lateralization performance was not limited by tone detection and to reduce effects of frequency selectivity. The actual noise levels were as follows: dioticHi: $10 \mathrm{~dB}$ below masked threshold, for both tone levels; dioticLo: $40 \mathrm{~dB}$ below masked threshold for the 70- $\mathrm{dB}$ tones and $25 \mathrm{~dB}$ below masked threshold for the 35- $\mathrm{dB}$ tones; and dichotic: $20 \mathrm{~dB}$ below masked threshold for the $70-\mathrm{dB}$ tones and $15 \mathrm{~dB}$ below masked threshold for the $35-\mathrm{dB}$ tones.

A two-interval, two-alternative, forced-choice (2I2AFC) weighted up-down method was used to track $75 \%$ correct lateralization. The first interval always contained the zero IPD reference tone while the second interval contained the tone, which was randomly lateralized to the left or right side. Listeners were instructed to indicate the direction of motion. The IPD was tracked logarithmically and the maximum IPD was restricted to $90^{\circ}$, since the extent of lateralization starts to decline for values above $90^{\circ}$ (Kunov and Abel, 1981). The background interferer was presented continuously during the whole run. A run was terminated after 14 reversals and the threshold was defined as the geometric mean of all IPD values following the fourth reversal. Listeners were trained in at least two sessions and performed more than 1200 lateralization judgments (constant stimuli) prior to actual data collection. IPD threshold was estimated as the geometric mean over three runs. If the SD over these runs, relative to the mean IPD threshold, exceeded a factor of 0.2 (which corresponds to a constant criterion in logarithmic units), additional runs were taken and the average of all was used. The final relative standard error of the IPD threshold estimate, averaged across all listeners and conditions, was 0.13 .

\section{B. Results and discussion}

The analysis of the lateralization results was performed on the log-transformed IPDs, as these satisfied the requirements of normal error distributions. This is in line with previous reports in literature on lateralization (e.g., Saberi, 1995; Lacher-Fougère and Demany, 2005). Figure 4 shows the IPD thresholds for the NH, OD, and HI listeners. The HI subjects $\mathrm{HI}_{7}$ and $\mathrm{HI}_{10}$ (not shown) performed much more poorly on lateralization than the remaining HI listeners. Therefore, their IPD thresholds were not included in the group averages and will be discussed separately further below. However, the conclusions presented in the following would remain unchanged if they were taken into account. Two trends can be seen in Fig. 4. First, lateralization performance was better at the higher tone level than at the lower level. Second, the HI listeners showed generally higher IPD thresholds than the NH listeners.

An ANOVA confirmed both the significant difference between $\mathrm{NH}$ and $\mathrm{HI}$ listeners $[F(1,12)=8.7, p=0.01]$, and the effect of tone level $[F(1,94)=71.5, p<0.0001]$. The effect of interferer condition was also significant $[F(3,94)$ $=27.8, p<0.0001]$, while interactions did not reach significance. The dichotic noise conditions led to the highest IPD 


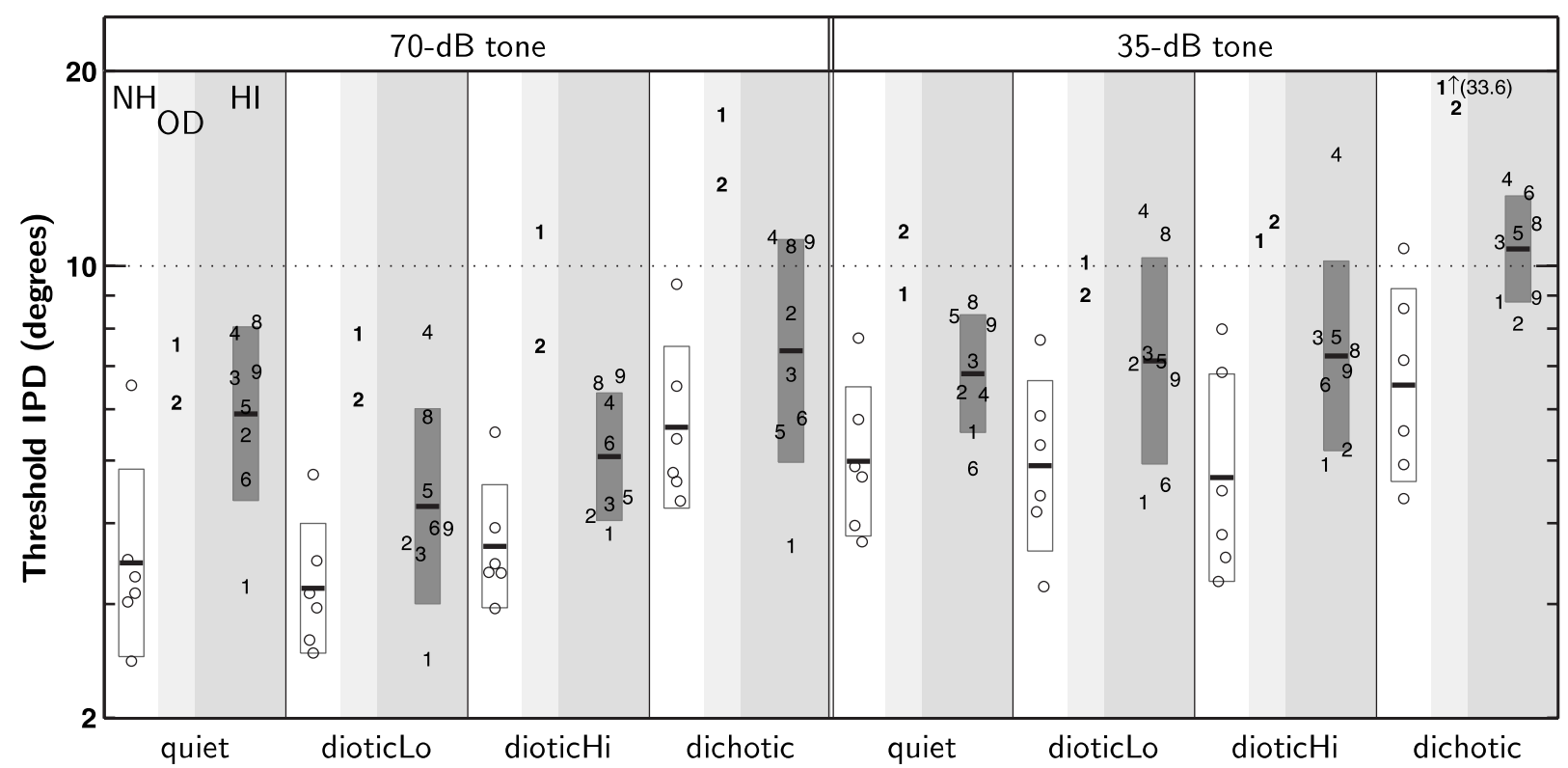

FIG. 4. Lateralization thresholds for the NH listeners (circles), the two listeners with OD (bold numbers), and the HI listeners (plain numbers), at two different tone levels (70 and $35 \mathrm{~dB}$ SPL) and for different interferer conditions (see text). Otherwise as Fig. 1.

thresholds, although the noise levels were actually lower than in the dioticHi conditions. This may, at least partly, be attributed to the fact that the dichotic noise gave rise to a diffuse, broad percept, while the diotic noise was lateralized in the midline. Hence, the latter provided an additional ongoing reference cue since the noise was switched on continuously during a run.

Comparing performance in the dioticLo and dioticHi conditions, the HI listeners seemed to cope as well as the $\mathrm{NH}$ listeners with the increase in noise level. Generally, noise did not have a greater effect on lateralization performance for the HI listeners than for the NH listeners, irrespective of tone and noise levels (as reflected in a lack of interaction between listener group and condition). Apart from higher thresholds for the HI listeners, the two groups of listeners showed a very similar pattern of results across conditions, with one exception, the quiet condition at the high tone level (leftmost panel in Fig. 4). Here, the lateralization thresholds for the HI listeners were a factor of 1.7 higher than for the NH listeners, while in the other conditions thresholds were, on average, a factor of 1.4 higher. For the dichotic condition at the same tone level (factor of 1.3), one might have expected a larger deficit than in the quiet condition: While in both conditions an ongoing reference cue was absent, a smaller fraction of nerve impulses would have been expected to be phase locked to the tone in the presence of the noise interferer, thus possibly producing more difficulties for the impaired auditory system. This was, however, not the case. Also, the HI listeners' deficit in quiet was actually smaller at the lower tone level (factor of 1.4) than at the higher level.

Hawkins and Wightman (1980) and Smoski and Trahiotis (1986) reported different effects of stimulus level on lateralization performance. For HI listeners with similar audiograms as in the present study, they measured lateralization thresholds in quiet at a low and a high stimulus level, in regions of normal hearing. For narrowband noise stimuli, the
HI listeners MM and MD in Hawkins and Wightman, (1980) showed a smaller lateralization deficit at the higher stimulus level than at the lower level. In contrast to this, and consistent with the present results, Smoski and Trahiotis (1986) observed a larger deficit in lateralization at the higher level using pure tones. In the same study, this trend was less clear when using narrowband noise stimuli. Hence, the discrepancy between the studies may be at least partly attributable to the differences in the stimuli.

Smoski and Trahiotis (1986) suggested that the lateralization judgment at high levels could be based on the excitation of a large portion of the basilar membrane rather than only on local excitation, and that a hearing loss might affect the integration of the non-local information. This interpretation is consistent with the present results for lateralization in quiet and in noise. At the tone level of $70 \mathrm{~dB}$ SPL, one would expect a substantial spread of excitation, particularly toward places that correspond to higher characteristic frequencies. The NH listeners might have integrated the additional information present at these high-frequency places, whereas the HI listeners might not have been able to benefit from this information, as it fell in the sloping region of their hearing loss. Indeed, if actually included, information from defective neural units (as, e.g., desynchronized information across frequencies) might have had a detrimental effect on lateralization acuity. The role of spread of excitation is reduced at the lower tone level of $35 \mathrm{~dB}$, but also at the higher level of $70 \mathrm{~dB}$ in the presence of background noise, as the latter partly masks non-local excitation. This would explain why the deficit observed for the HI listeners (relative to $\mathrm{NH}$ ) was largest at the high tone level in quiet.

As mentioned above, the $\mathrm{HI}$ subjects $\mathrm{HI}_{7}$ and $\mathrm{HI}_{10}$ performed more poorly on lateralization than the remaining $\mathrm{HI}$ listeners. Subject $\mathrm{HI}_{7}$ showed markedly increased lateralization thresholds, independent of interferer condition and tone level. His IPD thresholds ranged from $21^{\circ}$ to $27^{\circ}$ at the high 
tone level, and from $32^{\circ}$ to $40^{\circ}$ at the low tone level, without showing a particular susceptibility to noise interference. For subject $\mathrm{HI}_{10}$, lateralization thresholds could not be determined. Even after a considerable amount of training, her performance remained at chance level (even at the maximum IPD of $\left.90^{\circ}\right)^{2}$

The two OD listeners showed markedly higher lateralization thresholds than the $\mathrm{NH}$ listeners, for all interferer conditions and at both tone levels (see Fig. 4). On average, the IPD thresholds for subjects $\mathrm{OD}_{1}$ and $\mathrm{OD}_{2}$ were increased relative to those for the NH listeners, by factors of 2.6 and 2.2 , respectively. Both showed the most pronounced problems with lateralization in the presence of the dioticHi and dichotic noise interferers. In fact, in these conditions, they performed even more poorly than most of the HI listeners.

\section{FREQUENCY MODULATION DETECTION}

\section{A. Method}

FMDTs were measured monaurally for carrier frequencies of 125, 750, and $1500 \mathrm{~Hz}$. Prior to gating, the stimulus was a frequency-modulated sinusoid defined by

$$
s(t)=a \sin \left[2 \pi f_{c} t+\frac{\Delta f}{f_{m}} \sin \left(2 \pi f_{m} t+\varphi\right)\right],
$$

where $f_{c}$ represents the carrier frequency, $\Delta f$ represents the maximum frequency excursion, and $f_{m}$ represents the FM rate. The FM phase $\varphi$ was always $1.5 \pi$. The phase-lockingbased temporal mechanism for FM detection has been found to be operative only at FM rates below $10 \mathrm{~Hz}$, whereas at higher rates, FM detection is thought to be based primarily on a FM-to-AM conversion mechanism (e.g., Moore and Sek, 1996; Lacher-Fougère and Demany, 1998). Here, both mechanisms were tested, by using FM rates of 2 and $16 \mathrm{~Hz}$. The tone levels were $30 \mathrm{~dB}$ sensation level (SL; individual hearing thresholds were determined by means of 3I-3AFC detection measurements) and $70 \mathrm{~dB}$ SPL. The impact of noise interference was tested by measuring the FMDT for $2-\mathrm{Hz} \mathrm{FM}$ tones at $750 \mathrm{~Hz}$ in a bandlimited noise $(50-1500 \mathrm{~Hz})$, at a level $10 \mathrm{~dB}$ below the individual masked threshold. At $1500 \mathrm{~Hz}$, all measurements were undertaken in the presence of a low-level noise background $(50-3000 \mathrm{~Hz}$, with a spectrum level of $55 \mathrm{~dB}$ below the tone level), in order to mask low-frequency cues due to spread of excitation.

Finally, in order to assess the phase-locking-based mechanism further, similar to the paradigm used by Moore and Sek (1996), FMDTs for 2-Hz FM tones with a superimposed AM were measured at the carrier frequencies of 750 and $1500 \mathrm{~Hz}$. In view of the findings of Grant (1987), who observed a significantly larger deficit in FM detection in HI listeners if the FM tones were randomly rather than sinusoidally amplitude modulated, here, a quasi-sinusoidal AM was used: While the modulation depth was fixed at a peak-tovalley ratio of $6 \mathrm{~dB}$, the instantaneous modulation rate either increased or decreased as a linear function of time. According to Moore and Sek (1996), the peak-to-valley ratio of 6 $\mathrm{dB}$ should be large enough to disrupt FM-to-AM conversion cues, but still small enough not to induce substantial level- related pitch shifts. Hence, for the conditions with added AM, the amplitude $a$ in Eq. (1) was time dependent,

$$
a(t) \propto 1+m \sin \left(2 \pi F_{a}(t)+\vartheta\right) .
$$

Here, $m$ represents the AM depth and $F_{a}(t)$ is the integral of the instantaneous modulation rate

$$
F_{a}(t)=\int_{0}^{t} d \tau\left(f_{1}+\frac{f_{2}-f_{1}}{T} \tau\right)
$$

with $T$ representing the tone duration. The initial and final modulation rates $f_{1}$ and $f_{2}$ were each chosen randomly out of the interval between 1 and $3 \mathrm{~Hz}$, under the constraint $\left|f_{2}-f_{1}\right|>1 \mathrm{~Hz}$. Also the AM phase $\vartheta$ was randomized. Independent of condition, the FM tones had a duration of $750 \mathrm{~ms}$ and were gated with 50-ms raised-cosine ramps.

A 3I-3AFC weighted up-down method was used to track $75 \%$ correct FM detection. In the conditions without AM, two of the intervals contained unmodulated tones, whereas the target interval contained the FM tone. In the conditions with added AM, all three intervals were independently amplitude modulated and the listeners were instructed to detect the interval containing the FM by listening for its characteristic high-low-high warble. The maximum frequency excursion $\Delta f$ was tracked logarithmically. A run was terminated after 12 reversals and the threshold was defined as the geometric mean of all $\Delta f$ values following the fourth reversal. Prior to data collection, a training session was given in which the listeners were trained on all conditions. Initially, both ears were tested on 2-Hz FM detection at $750 \mathrm{~Hz}$ in quiet and subsequently the worse ear was chosen for further testing. This was done in order to obtain the largest possible range of FMDTs among the HI listeners, particularly in view of the subsequent comparison with the results of the other tests such as frequency selectivity. Furthermore, it seemed reasonable to assume that the worse ear was limiting the binaural TFS-processing performance, particularly in the lateralization task. The FMDT was estimated as the geometric mean over three runs. If the SD over these runs, relative to the mean FMDT, exceeded a factor of 0.15 , additional runs were taken and the average of all was used. The final relative standard error of the FMDT estimate, averaged across all listeners and conditions, was 0.08 .

\section{B. Results and discussion}

The analysis of the FM detection results was performed on the log-transformed FMDTs, as these satisfied the requirements of normal error distributions. This is in agreement with previous reports in literature on FM detection (e.g., Zurek and Formby, 1981; Buss et al., 2004). For all listeners, FM detection performance did not differ significantly between the tone levels of $30 \mathrm{~dB}$ SL and $70 \mathrm{~dB}$ SPL (two-tailed t-test: $p=0.79$ ). Therefore, only the $30-\mathrm{dB}$ results are considered in the following. Figure 5 shows the FMDTs for the $\mathrm{NH}, \mathrm{OD}$, and $\mathrm{HI}$ listeners. As can be seen, for all groups, the FMDTs increased with increasing carrier frequency, consistent with previous studies (e.g., Demany and Semal, 1989). 


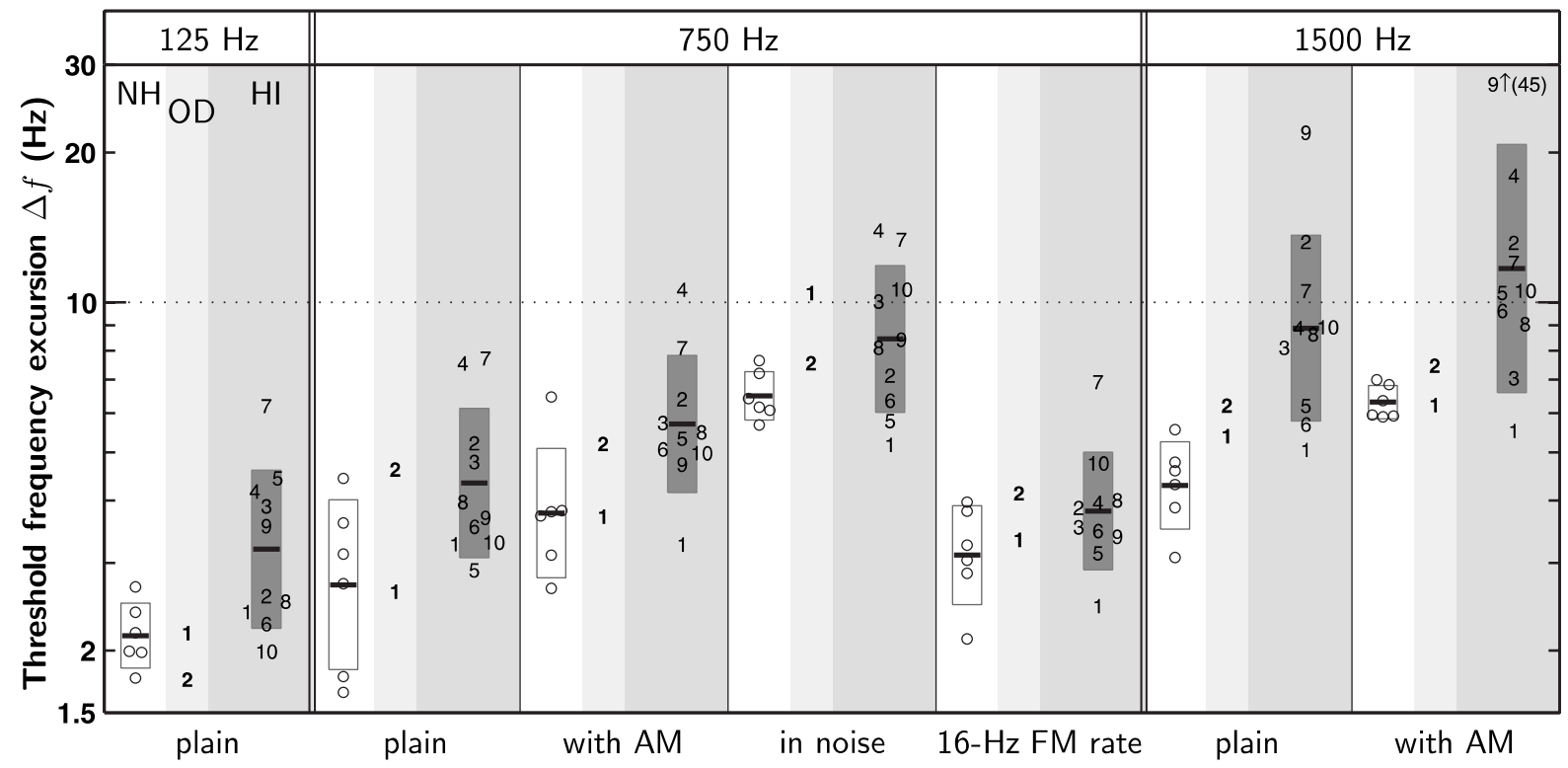

FIG. 5. FMDTs for the NH listeners (circles), the two listeners with OD (bold numbers), and the HI listeners (plain numbers), for three different carrier frequencies $(125,750$, and $1500 \mathrm{~Hz}$ ) and for different measurement conditions (see text; "plain" refers to 2-Hz FM in quiet). All results were obtained at $30 \mathrm{~dB}$ SL. Otherwise as Fig. 1.

The HI listeners performed generally more poorly than the NH listeners. On average, their FMDTs were a factor of 1.5 higher than for the NH listeners.

An ANOVA confirmed the statistical significance of the group difference $[F(1,14)=16.9, p=0.001]$ as well as the effect of tone frequency $[F(1,89)=56.7, p<0.0001]$. No significant interaction between listener group and tone frequency was observed $[p=0.19]$. While the log-transformed FMDTs increased linearly as a function of frequency, the Weber fractions $\Delta f / f_{c}$ decreased from 125 to $750 \mathrm{~Hz}$ by a factor of 4 and then remained constant up to $1500 \mathrm{~Hz}$. Zurek and Formby (1981) measured FMDTs in HI listeners and found larger deficits for low-frequency tones than for highfrequency tones, given the same degree of hearing loss $(<30 \mathrm{~dB}$ HL$)$ at the test frequency. However, the FM detection deficits at $125 \mathrm{~Hz}$ observed in the present study were substantially smaller than the ones reported in that study. This might be due to the fact that the HI listeners of Zurek and Formby (1981) showed slightly higher audiometric thresholds at $125 \mathrm{~Hz}$ and generally more severe losses below $1000 \mathrm{~Hz}$ than the HI listeners of the present study.

FMDTs differed significantly across measurement conditions [2-Hz FM in quiet ("plain"), added AM, noise interference, and higher FM rate; $F(3,89)=24.1, p<0.0001]$. The interaction between listener group and measurement condition reached only marginal significance $[F(3,89)$ $=2.5, p=0.07$. However, for the following multiple comparison analysis, the interaction term was kept in the mixedeffects model.

As revealed by the multiple comparisons, the group differences between $\mathrm{NH}$ and $\mathrm{HI}$ listeners were significant for the $2-\mathrm{Hz} \mathrm{FM}$ in quiet and the condition with added $\mathrm{AM}$ [group difference in terms of $\log _{10}$ (FMDTs) for $2-\mathrm{Hz}$ FM: 0.23 (CI 0.09, 0.37); group difference with added AM: 0.20 (CI 0.04, 0.35)]. For all listeners, the FMDTs with added AM were increased relative to those for the condition with FM only. However, as this increase was similar for the $\mathrm{NH}$ and $\mathrm{HI}$ listeners, it seems that both groups relied to a comparable extent on FM-to-AM conversion cues, when AM was absent. No significant group difference was found in the condition with the higher FM rate of $16 \mathrm{~Hz}$ [group difference: 0.09 (CI $-0.08,0.26)]$. Thus, regarding the different FM rates $(2 \mathrm{~Hz}$ vs $16 \mathrm{~Hz})$, the $\mathrm{HI}$ listeners showed a significant deficit on FM detection at the low rate but not at the high rate, where the FM-to-AM conversion is supposed to be the dominant detection mechanism. This can be seen in Fig. 5 (second and fifth panels): While the HI listeners' performance was better for the higher FM rate, the NH listeners' performance was worse. Taken together, this suggests that the observed deficits in the detection of $2-\mathrm{Hz}$ FM were indeed due to problems with phase-locking-based TFS processing.

In the presence of the noise interferer, all listeners performed worse than in quiet. However, the HI listeners did not perform significantly more poorly than the $\mathrm{NH}$ listeners in this condition [group difference: 0.11 (CI $-0.06,0.29)$ ]. Hence, the HI listeners did not show an increased susceptibility to noise interference. This is in agreement with the results of Turner (1987), who measured pure-tone frequency difference limens in the presence of low-frequency masking noise for four $\mathrm{NH}$ and four $\mathrm{HI}$ listeners and found a similar effect of the noise upon performance for the two groups of listeners. Also, Horst (1987) measured frequency discrimination in noise. However, the question of a different impact of noise on the performance of the $\mathrm{NH}$ and $\mathrm{HI}$ listeners could not be addressed, since he did not measure the frequency difference limen for a given noise level but determined the noise level at which a given fixed frequency difference could just be perceived.

Figure 5 also shows the FMDTs for the two OD listeners. Their FMDTs did not differ substantially from those for the $\mathrm{NH}$ listeners. Subject $\mathrm{OD}_{2}$ performed at the lower edge 


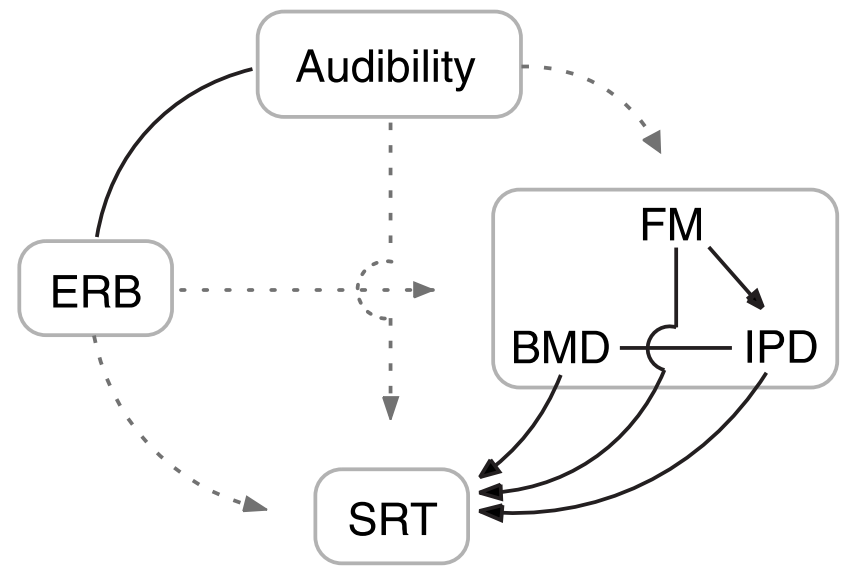

FIG. 6. Relations between the results for the different auditory tests within the group of HI listeners: pure-tone hearing thresholds (audibility), frequency selectivity (ERB), monaural frequency modulation detection (FM), binaural masked detection (BMD), tone lateralization (IPD), and speech reception (SRT). Solid lines indicate significant correlations whereas dotted lines indicate correlations that were not significant. The direction of the arrows is solely based on the assumed sequence of processing in the auditory pathway. Therefore, arrowheads were omitted where the order is uncertain or where the processing might take place in parallel rather than in sequence.

of the $\mathrm{NH}$ listeners except for the $125-\mathrm{Hz}$ carrier, where her performance was good. For subject $\mathrm{OD}_{1}$, a deficit was observed for the $750-\mathrm{Hz}$ carrier with interfering noise. Otherwise her performance was essentially normal.

\section{COMPARISON OF RESULTS ACROSS TESTS}

\section{A. Hearing-impaired listeners}

Pearson correlations and two-tailed $p$ values were examined to study the relations between the results of the different auditory tests within the group of HI listeners. The findings are schematized in Fig. 6.

\section{Correlations with absolute hearing thresholds}

Frequency selectivity in terms of the ERB at $750 \mathrm{~Hz}$ was significantly correlated with the individual hearing threshold at this frequency $[r=0.77, p=0.009]$. Here, the hearing threshold was estimated by means of a 3I-3AFC method with a 1-dB stepsize. When the standard audiometric threshold (with a 5-dB stepsize) was considered instead, the correlation was smaller [ $r=0.53]$, but increased when thresholds were averaged in terms of the pure-tone average (PTA) threshold at $0.5,1,2$, and $4 \mathrm{kHz}[r=0.8]$. The finding of a correlation between frequency selectivity and hearing threshold is consistent with previous reports in literature (e.g., Tyler et al., 1983; Moore, 1996), although less distinct correlations have been observed for hearing losses below $30 \mathrm{~dB}$ HL (see Baker and Rosen, 2002).

No significant correlations were observed between individual hearing thresholds and performance in the three tests of TFS processing (binaural masked detection, lateralization, and FM detection). Tones with equal sound pressure levels were used for all listeners in the masked detection and lateralization tasks. Hence, the deficits in performance that were observed at the low tone level of $35 \mathrm{~dB}$ SPL could have been due to the slightly differing sensation levels (ranging from 32 to $38 \mathrm{~dB}$ SL for the $\mathrm{NH}$ group and from 23 to $34 \mathrm{~dB}$ SL for the HI group). However, the absence of correlations between hearing thresholds, and thereby sensation levels, and masked detection/lateralization performance makes this unlikely. With regard to FM detection, subject $\mathrm{HI}_{9}$, who showed markedly worse performance at $1.5 \mathrm{kHz}$, also had the highest hearing thresholds at this frequency. Nevertheless, the correlation between the hearing thresholds and FMDTs at $1.5 \mathrm{kHz}$ was not significant when considering all HI listeners $[r=0.39, p=0.27]$. Finally, the hearing thresholds were not significantly correlated with the results for speech reception, regardless of whether the hearing thresholds at single frequencies or averages across frequencies were considered.

The absence of correlations with the hearing thresholds can, to some extent, be attributed to the homogeneity of the HI group in terms of their audiograms. Also, given the limited number of listeners, only rather strong correlations would be expected to be significant. Hence, here and in the following, the absence of a significant correlation does not necessarily imply the absence of a relationship.

\section{Correlations between the various tests of TFS processing and frequency selectivity}

The deficits observed for the HI listeners with binaural masked detection, lateralization, and FM detection provide strong evidence for deficits with phase-locking-based TFS processing. However, no significant correlations were observed between frequency selectivity and these tests of TFS processing. ${ }^{3}$ This can be illustrated by means of individual results among the HI listeners: Subject $\mathrm{HI}_{1}$ showed poor frequency selectivity but good TFS-processing skills, whereas subject $\mathrm{HI}_{7}$ performed well on the former but poorly on the latter. Subject $\mathrm{HI}_{10}$ showed poor performance in both domains. Hence, it seems that the deficits found in TFS processing cannot be attributed solely to a deficit in frequency selectivity, but must be, at least partly, due to another impairment factor. This is further supported by the finding of TFSprocessing deficits in quiet, which cannot be explained in terms of frequency selectivity.

Significant correlations were found among the tests of TFS processing. When correlations between the tests were observed for multiple test conditions, such as for the different interferer conditions in the lateralization task, an overall correlation is given in the following, instead of reporting the correlations for each individual condition. The overall correlation is based on the listeners' average performance on that test. This average performance was measured in terms of the estimated random effect, which summarizes individual performance across multiple conditions. Here, it represents the performance deviation of an individual $\mathrm{HI}$ listener from the HI group mean. Since the random effect accounts for multiple measurement conditions simultaneously, the corresponding correlation results are more robust and more conservative in terms of significance. Using this statistic, a significant correlation was observed between lateralization performance and the binaural masked thresholds in the $N_{0} S_{\pi}$ condition $[r=0.80, p=0.01]$, as has been observed previously (Hall et al., 1984; Kinkel et al., 1988; Koehnke et al., 
1995). While the correlation between lateralization performance and the $N_{u} S_{0}$ thresholds was rather marginal [ $p \sim 0.08]$, no such correlation was observed for the $N_{0} S_{0}$ thresholds $[p>0.2]{ }^{4}$ The above correlation between lateralization performance and $N_{0} S_{\pi}$ detection thresholds remained significant when controlling for individual hearing thresholds by means of partial correlation $[r=0.83, p=0.01]$.

Performance on monaural FM detection and binaural masked detection was not correlated significantly. ${ }^{5}$ However, the monaural FMDTs at $750 \mathrm{~Hz}$ were significantly correlated with lateralization performance $[r=0.79, p=0.01]$. Considering the different FM conditions separately, the correlations were strongest for the conditions with noise interference and with added AM. The correlation remained significant when controlling for individual hearing thresholds by means of partial correlation $[r=0.79, p=0.02]$. The fact that binaural and monaural (suprathreshold) TFS processing were correlated for the HI listeners suggests that the binaural deficit might be mainly attributable to a monaural impairment factor.

\section{Correlations with speech reception}

As depicted in Fig. 7, two of the full-spectrum speech conditions, LATSSN and TWOTALK, showed significant correlations with the measures of TFS processing while no significant correlations were observed for the other speech conditions, including filtered speech. ${ }^{6}$ Performance in the dichotic masked detection tasks (conditions $N_{0} S_{\pi}$ and $N_{u} S_{0}$, in terms of the estimated random effects) was correlated with the SRTs in the LATSSN condition $[r=0.85, p=0.002]$; see Fig. 7(a). The correlation was also significant when the masking release instead of the SRT was considered $[r=0.80, p=0.005]$. For the sake of brevity in the following, a correlation with the masking release will only be given if it was stronger than the correlation with the corresponding SRT itself. The SRTs in the LATSSN condition were also significantly correlated with lateralization performance, but only for the dioticHi condition at the high tone level $[r=0.80, p=0.02]$; see Fig. $7(\mathrm{~b}){ }^{7}$ The pattern of correlations between the LATSSN SRTs and the masked thresholds as well as the lateralization thresholds remained unchanged when partialing out the individual hearing thresholds [masked detection: $r=0.82, p=0.007$; lateralization: $r=0.76, p<0.05]$. For the TWOTALK condition, significant correlations were found with both the dichotic masked thresholds $\left(N_{0} S_{\pi}\right.$ and $\left.N_{u} S_{0}\right)$ and the lateralization thresholds in the dioticHi condition [masked detection: $r=0.68$, $p=0.03$; lateralization: $r=0.84, p=0.009]$, as can be seen in Figs. 7(c) and 7(d), respectively. While the correlation with the masked thresholds was marginal when controlling for the individual hearing thresholds, the correlation with the lateralization thresholds remained significant [masked detection: $r=0.60, p=0.09$; lateralization: $r=0.81, p=0.03]$.

No significant correlations between performance on speech reception and FMDTs at 125 and $750 \mathrm{~Hz}$ were found. However, at $1.5 \mathrm{kHz}$, the FMDTs with added AM were significantly correlated with the SRT in the TWOTALK condition $[r=0.75, p=0.013]$. Here, the correlation was stronger for the corresponding masking release $[r=-0.77$,
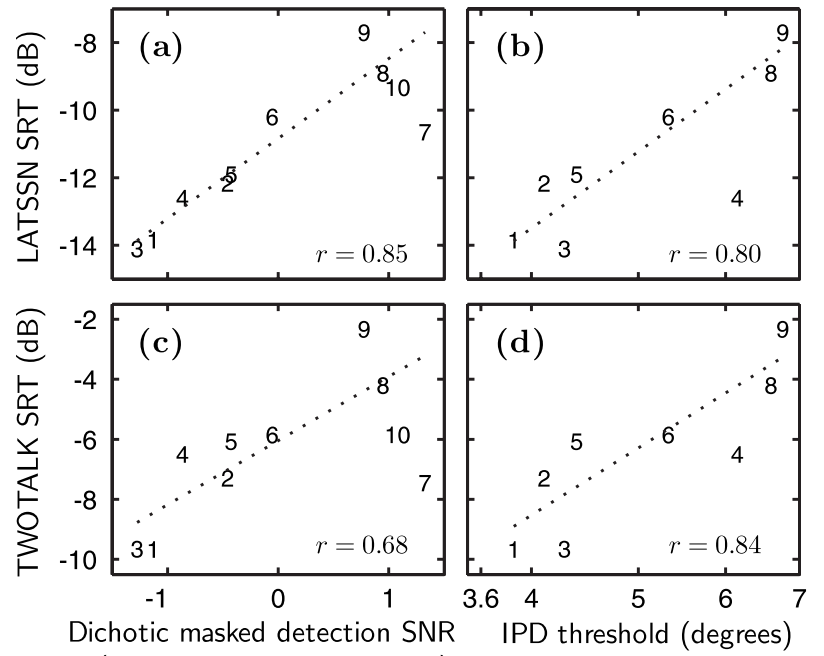

(standardized random effect)

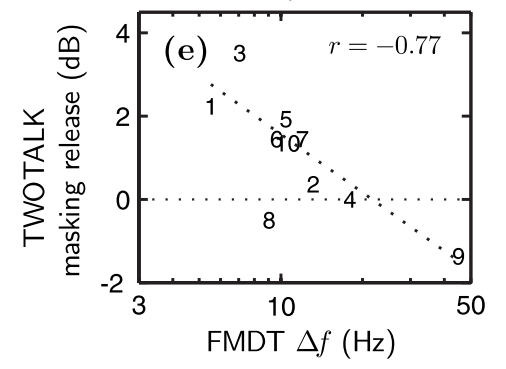

FIG. 7. Correlations between performance on speech reception and TFS processing within the group of HI listeners. The dotted regression lines were obtained by means of least trimmed squares robust regression. (a) Correlation between the LATSSN SRTs and performance for dichotic masked detection $\left(N_{u} S_{0}\right.$ and $N_{0} S_{\pi}$ conditions). The latter is given in terms of the standardized random effects, which measure the individual deviations from the HI group mean. Better/worse than average performance, i.e., a smaller/larger threshold SNR, results in a negative/positive random effect. The interval from -1 to 1 covers $68 \%$ of the HI "population." (b) Correlation between the LATSSN SRTs and the IPD thresholds in the dioticHi condition for the 70-dB tones. [(c) and (d)] Same as (a) and (b) but for TWOTALK SRTs. (e) Correlation between the TWOTALK masking release (re SSN) and the FMDTs for $1.5-\mathrm{kHz}$ tones with added AM.

$p=0.009$ ], as depicted in Fig. 7(e). When controlling for the individual hearing thresholds at $1.5 \mathrm{kHz}$, the correlation with the SRT was marginal, while the correlation with the masking release remained significant [SRT: $r=0.61, p=0.08$; masking release: $r=-0.67, p<0.05]$. Generally, the observed correlations were only slightly affected when the effect of absolute hearing thresholds was partialed out. To some degree, this can be attributed to the homogeneity of the HI group in terms of their hearing thresholds.

The finding of a correlation between the SRTs for the dichotic LATSSN masker and binaural low-frequency TFS processing seems reasonable in view of the results reported by Schubert and Schultz (1962) and Levitt and Rabiner (1967). They found that the release from masking for dichotic speech in noise $\left(N_{0} S_{0.5 \mathrm{~ms}}\right.$ or $\left.N_{0} S_{\pi}\right)$ was primarily determined by interaural time or phase disparity at low frequencies. Besides, in the present study, binaural masked detection and dichotic speech reception depended in the same way on binaural integration: While they could be accomplished monaurally, use of the binaural information would give rise to better performance. While the LATSSN 
condition assessed the ability to take advantage of an interaural timing mismatch between target speech and noise interferer, performance in the TWOTALK background depended particularly on the ability to separate the target talker and the two interfering talkers. Hence, the correlations found between the SRTs for TWOTALK background and the measures of TFS processing support the hypothesis of Zeng et al. (2005) that TFS cues might be utilized in talker separation in order to improve performance in listening situations with competing talkers. In this respect, the correlation between speech reception in the TWOTALK background (in terms of SRT and masking release) and FM detection performance at $1.5 \mathrm{kHz}$, observed here, may indicate a potential contribution of the second formant region (cf. Peterson and Barney, 1952) to talker identification and separation.

TFS processing was not correlated with SRTs (or masking releases) in the fluctuating backgrounds, SAM and RAM, neither for full-spectrum nor filtered speech. Hence, in contrast to Lorenzi et al., (2006), no evidence was found for a relation between TFS processing and dip listening. This discrepancy might have been due to the fact that the HI listeners in Lorenzi et al., (2006) had "flat" moderate hearing losses ( $\sim 50 \mathrm{~dB} \mathrm{HL})$, whereas the HI listeners in the present study had "normal" hearing thresholds up to $1 \mathrm{kHz}$. Furthermore, Lorenzi et al. (2006) tested TFS processing with processed speech stimuli, which exhibited more complex TFS patterns than the tone stimuli used in the present study (with the exception of the uncorrelated noise maskers in the $N_{u} S_{0}$ masked detection task).

A correlation between frequency selectivity and speech reception, as previously reported in literature (e.g., Dreschler and Plomp, 1985; Horst, 1987), was not observed here. However, these studies often included estimates of frequency selectivity at frequencies above $1000 \mathrm{~Hz}$, while, in the present study, frequency selectivity was estimated only at $750 \mathrm{~Hz}$. This may explain the absence of a correlation in the case of the full-spectrum speech, but not for the lowpass-filtered speech. Another possible explanation, which might also account for the results with filtered speech, is that several impairment factors contributed to the observed speech reception deficits in complementary ways. Indeed, when the lowfrequency slopes of the estimated filters and the monaural FMDTs at $1.5 \mathrm{kHz}$ (with added AM) were considered as joint predictors in a multiple regression analysis, their combined effect on the monaural SRTs in the SSN and SAM conditions was significant [combined effect of filter slope and FMDT for $\operatorname{SSN}: F(2,7)=9.6, p=0.01$; for $\operatorname{SAM}: F(2,7)=8.5$, $p=0.01]$. The combined effect was less significant when the ERB instead of the filter slope was considered (for SSN: $p=0.04$; for SAM: $p=0.05$ ). However, regression results that rely on such conjunctions of variables, rather than on strong primary correlations, should be viewed with caution, particularly in view of the small number of subjects.

\section{Possible relations to aging}

One concern is that the $\mathrm{NH}$ listeners in the present study were, on average, younger than the HI listeners (median age 28 and 63 years, respectively). This raises the question of possible age effects, as previous studies have suggested a relation between aging and deficits in TFS processing as well as speech reception (e.g., Pichora-Fuller and Schneider, 1992; Strouse et al., 1998; Schneider et al., 2002; Ross et al., 2007). Indeed, subject $\mathrm{HI}_{1}$, who was the youngest of the $\mathrm{HI}$ listeners, performed better than the other HI listeners on the three tests of TFS processing, particularly lateralization and FM detection. However, apart from her age, $\mathrm{HI}_{1}$ also differed in terms of etiology, as her hearing loss was due to hypoxia at birth. For the remaining HI listeners (53-74 years), dichotic masked detection was significantly correlated with age, while results for the other TFS tests were not (dichotic masked detection: $r=0.81, p=0.01$; lateralization: $r=0.36, p=0.37$; FM detection: $r=0.13, p=0.75$ ). Hence, it cannot be excluded that part of the TFS deficits observed for the HI listeners could be related to aging. Ross et al. (2007) recorded cortical auditory-evoked responses to tones with dynamic changes in IPD. They found that the highest carrier frequency, at which responses to changes in IPD could be detected, declined with age. This indicates that aging might induce or potentiate a degradation in the processing of TFS at a peripheral or central auditory level, which is not reflected in the pure-tone hearing thresholds.

\section{B. Listeners with obscure dysfunction}

The two OD listeners showed deficits in frequency selectivity and binaural masked detection, which were comparable to those of the HI listeners. In the lateralization task they performed even more poorly than most of the HI listeners, showing substantial deficits, particularly with lateralization in background noise. However, in contrast to the HI listeners, who showed similar deficits on binaural lateralization and monaural FM detection, the OD listeners did not show as clear deficits in the FM detection task as in the lateralization task. Since FM detection was assessed monaurally, one might conjecture that it was the non-tested ear that was actually limiting the lateralization performance. However, this can be excluded, as both ears were screened initially on FM detection and the worse ear was chosen for further testing. A possible reason for the poor binaural TFS performance of the OD listeners could be the large bandwidth differences between their ears. Colburn and Häusler (1980) suggested that the output of differing filters, given a diotic wideband input signal, would be partly uncorrelated at the two ears, resulting in lateralization blur. However, this explanation does not account for the observed poor performance in quiet and in dichotic (uncorrelated) noise. Hence, it seems that the TFS processing was affected at the stage of binaural integration rather than at a preceding monaural stage. Alternatively, even if the binaural TFS information was accurately integrated, it might not have been accessible at following stages of auditory processing.

The OD listeners showed rather small deficits in the reception of full-spectrum speech, but clear deficits in the reception of lowpass-filtered speech. These deficits might, at least partly, be attributable to the deficits in frequency selectivity and binaural TFS processing, which were observed to a similar extent for both OD listeners. However, additional personality-related factors, such as an individual's underesti- 
mation of their own hearing ability (lack of "auditory confidence"), may be involved in the phenomenon of obscure (auditory) dysfunction. Considering the heterogeneity of the clinical group of OD patients (e.g., Saunders and Haggard, 1989; Zhao and Stephens, 2000) and the fact that the diagnosis of OD is solely based on a self-rated disability, the necessity for such factors is almost self-evident.

\section{POSSIBLE UNDERLYING IMPAIRMENT MECHANISMS}

Figure 6 illustrates that TFS processing was related neither to audibility nor to frequency selectivity, although deficits were found in all of the tests. One may speculate about possible impairment sites and mechanisms underlying these deficits. Damage to or loss of OHCs has been shown to result in a loss of sensitivity and frequency selectivity (e.g., Evans and Harrison, 1976; Liberman and Dodds, 1984), while damage to or loss of inner hair cells (IHCs) does not seem to have any substantial effect on sensitivity or tuning of the remaining intact IHCs (e.g., Wang et al., 1997). Hence, OHC damage might have been responsible for the deficits in frequency selectivity and their relation to absolute threshold observed here (cf. Moore et al., 1999).

Several factors might have contributed to the deficits in TFS processing. A loss of OHCs could have resulted in a reduced precision of phase locking (Woolf et al., 1981). However, this is controversial, as other studies did not find evidence for such phase-locking anomalies (e.g., Miller et al., 1997). Apart from this, Woolf et al. (1981) found the reduced phase locking to be related to elevated absolute thresholds, which was not observed for the TFS deficits in the present study. Also, a loss of OHCs might have altered the spatiotemporal response pattern of the basilar membrane. This could have affected TFS processing if TFS information was extracted by cross-correlation of the outputs of different places along the basilar membrane (e.g., Deng and Geisler, 1987; Shamma, 2001; Carney et al., 2002). Since the present study assessed $\mathrm{OHC}$ integrity in terms of frequency selectivity only at a single frequency, this option cannot be ruled out here.

Alternatively, through partial section of the auditory nerve, it has been shown that a loss of auditory-nerve fibers of up to $90 \%$ does not necessarily result in elevated puretone thresholds (e.g., Schuknecht and Woellner, 1953). Hence, the observed TFS deficits in regions of normal hearing might be attributable to damage to or loss of auditorynerve fibers or the innervated IHCs. A related possibility concerns the (monaural) enhancement of phase-locking synchrony to low-frequency tones that has been observed in the cochlear nucleus (e.g., Joris et al., 1994) and might be reduced in impaired hearing. The alternative possibility, however, that a specific binaural processing stage, such as interaural coincidence detection, was affected in the HI listeners seems implausible given the clear correlation between the monaural and binaural TFS deficits found in these listeners.

\section{SUMMARY AND CONCLUSIONS}

In addition to deficits in speech reception, deficits in frequency selectivity and in phase-locking-based TFS processing were observed for HI listeners, despite testing in regions of normal hearing. The observed TFS deficits were not related to reduced frequency selectivity. Monaural and binaural TFS deficits, however, were found to be related, suggesting that the binaural deficits might have been attributable to a monaural impairment factor. Background noise did not have a larger effect on TFS processing for the HI listeners than for the NH listeners: Although the acuity of TFS processing was decreased for the $\mathrm{HI}$ listeners, it seemed to be as robust to noise interference as for the NH listeners. SRTs in a two-talker background and in lateralized noise, but not in amplitude-modulated noise, were correlated with TFSprocessing performance, suggesting that TFS information might be utilized in talker separation and spatial segregation.

The OD listeners showed deficits in frequency selectivity and in binaural, but not monaural, TFS processing. Compared with the NH listeners, their SRTs were particularly elevated for lowpass-filtered speech.

These findings on auditory deficits, as well as preserved auditory abilities, may serve as constraints for future models of the impaired auditory system. Furthermore, they may help in defining an auditory profile for listeners with impaired hearing.

\section{ACKNOWLEDGMENTS}

We wish to thank Graham Naylor, Christian Lorenzi, Steven Greenberg, Eric R. Thompson, and our colleagues at the Centre for Applied Hearing Research for valuable comments and stimulating discussions. We also thank Brian C. J. Moore and three anonymous reviewers for their constructive criticism on an earlier draft of this paper. We are grateful to all the listeners for their participation in many hours of testing. This work was supported by the Danish Research Foundation, the Danish Graduate School SNAK "Sense organs, neural networks, behavior, and communication," and the Oticon Foundation.

${ }^{1}$ In addition to the $\operatorname{roex}(p, r)$ filter model, the more complex variants
$\operatorname{roex}(p, w, t)$ and $\operatorname{roex}(p, w, t, p)$, as given in Oxenham and Shera $(2003)$,
were fitted to the threshold data. However, the gain in terms of goodness
of fit was negligible, with an average change in the rms error by a factor
of 0.96 and a maximum change by a factor of 0.7 in a single case. This is
in contrast to Oxenham and Shera $(2003)$, who found a considerably larger
reduction in the rms error. The discrepancy may be due to the use of a
short signal duration ( $10 \mathrm{ms)}$ and low sensation levels (10 to 35 dB SL) in
their study. Since the results obtained here with the more complex filter
models were very similar to those obtained with the simple roex $(p, r)$
model, the discussion was limited to the latter.
${ }^{2}$ While subject HI showed consistently poor performance on all TFS-
processing tests (poorest performance of all listeners on binaural masked
detection and FM detection), subject HI ${ }_{10}$, who was not able to lateralize
at all, showed relatively poor performance on masked detection, but aver-
age performance in the FM detection task. Although it was ensured that
HI ${ }_{10}$ had understood the lateralization task, it cannot be excluded that her
problem was, at least partly, due to the nature of the $2 \mathrm{I}-2 \mathrm{AFC}$ task, rather
than a problem with lateralization per se.
${ }^{3}$ At first, it may seem surprising that the diotic masked thresholds ( $\left.N_{0} S_{0}\right)$
and frequency selectivity were not correlated. However, in addition to the O. Strelcyk and T. Dau: Relations between impaired auditory functions 
filter bandwidth, the masked thresholds are determined by the detector efficiency, i.e., the SNR at the output of the auditory filter required for detection.

${ }^{4}$ The fact that no significant correlation was observed between the IPD thresholds and the $N_{0} S_{0}$ or $N_{u} S_{0}$ masked thresholds is not surprising, as the levels of the diotic and dichotic noise interferers in the lateralization task had been chosen according to the individual $N_{0} S_{0}$ and $N_{u} S_{0}$ detection thresholds, in order to make sure that lateralization performance was not limited by tone detection.

${ }^{5} \mathrm{~A}$ reason for this could be that FM detection constituted a suprathreshold measure of TFS processing, while masked detection assessed the latter at threshold. Apart from this, since the tone detection could have been accomplished monaurally, it seems reasonable to assume that the binaural detection performance was not solely determined by the "worse" ear, which was tested on FM detection.

${ }^{6}$ In the filtered-speech conditions, listeners $\mathrm{HI}_{6}$ and $\mathrm{HI}_{9}$ performed markedly more poorly than all other listeners (Fig. 1). Subject $\mathrm{HI}_{9}$ showed the largest deficits in speech reception among the HI listeners. However, his poor performance on $\mathrm{FM}$ detection at $1.5 \mathrm{kHz}$, which might have been a sign of substantial deficits in the processing of high-frequency information, cannot account for the deficits in the reception of lowpass-filtered speech. Similarly, subject $\mathrm{HI}_{6}$ 's problems with lowpass-filtered speech were not reflected in his performance on the auditory tests of frequency selectivity or TFS processing. The reason for this remains unclear.

${ }^{7}$ Given that a dichotic noise interferer was used in the LATSSN speech condition, it might seem counterintuitive that a correlation was found in the case of the dioticHi lateralization condition but not the dichotic condition. However, the dichotic noise interferer (as compared to a diotic one) exerted rather opposite effects on speech reception and lateralization: While it gave rise to a release from masking in the speech task, it represented an additional challenge in the lateralization task. Furthermore, the level of the dioticHi noise in the lateralization task was comparable to the level of the noise interferer in the speech task (if the level is considered relative to the corresponding masked threshold for tone and speech, respectively).

Abbas, P. J. (1981). "Auditory-nerve fiber responses to tones in a noise masker," Hear. Res. 5, 69-80.

Baker, R. J., and Rosen, S. (2002). "Auditory filter nonlinearity in mild/ moderate hearing impairment," J. Acoust. Soc. Am. 111, 1330-1339.

Buss, E., Hall, J. W., and Grose, J. H. (2004). "Temporal fine-structure cues to speech and pure tone modulation in observers with sensorineural hearing loss," Ear Hear. 25, 242-250.

Carney, L. H., Heinz, M. G., Evilsizer, M. E., Gilkey, R. H., and Colburn, H. S. (2002). "Auditory phase opponency: A temporal model for masked detection at low frequencies," Acta. Acust. Acust. 88, 334-347.

Colburn, H., and Häusler, R. (1980). "Note on the modeling of binaural interaction in impaired auditory systems," in Psychophysical, Physiological, and Behavioral Studies in Hearing, edited by G. van den Brink and F. A. Bilsen (Delft University Press, Delft).

Colburn, H. S. (1996). "Computational models of binaural processing," in Auditory Computation, edited by H. Hawkins, T. McMullen, A. Popper, and R. Fay (Springer-Verlag, New York).

Costalupes, J. A. (1985). "Representation of tones in noise in the responses of auditory nerve fibers in cats. I. Comparison with detection thresholds," J. Neurosci. 5, 3261-3269.

Demany, L., and Semal, C. (1989). "Detection thresholds for sinusoidal frequency modulation," J. Acoust. Soc. Am. 85, 1295-1301.

Deng, L., and Geisler, C. D. (1987). "A composite auditory model for processing speech sounds," J. Acoust. Soc. Am. 82, 2001-2012.

Dreschler, W. A., and Plomp, R. (1985). "Relations between psychophysical data and speech perception for hearing-impaired subjects. II," J. Acoust. Soc. Am. 78, 1261-1270.

Durlach, N. I., Thompson, C. L., and Colburn, H. S. (1981). "Binaural interaction in impaired listeners. A review of past research," Audiology 20, 181-211.

Evans, E. F., and Harrison, R. V. (1976). "Correlation between cochlear outer hair cell damage and deterioration of cochlear nerve tuning properties in the guinea-pig," J. Physiol. 256, 43P-44P.

Festen, J. M., and Plomp, R. (1983). "Relations between auditory functions in impaired hearing," J. Acoust. Soc. Am. 73, 652-662.

Freyman, R. L., and Nelson, D. A. (1991). "Frequency discrimination as a function of signal frequency and level in normal-hearing and hearingimpaired listeners," J. Speech Hear. Res. 34, 1371-1386.
Füllgrabe, C., Berthommier, F., and Lorenzi, C. (2006). "Masking release for consonant features in temporally fluctuating background noise," Hear. Res. 211, 74-84.

Gabriel, K. J., Koehnke, J., and Colburn, H. S. (1992). "Frequency dependence of binaural performance in listeners with impaired binaural hearing," J. Acoust. Soc. Am. 91, 336-347.

Gilbert, G., and Lorenzi, C. (2006). "The ability of listeners to use recovered envelope cues from speech fine structure," J. Acoust. Soc. Am. 119, $2438-$ 2444.

Glasberg, B. R., and Moore, B. C. J. (1989). "Psychoacoustic abilities of subjects with unilateral and bilateral cochlear hearing impairments and their relationship to the ability to understand speech," Scand. Audiol. Suppl. 32, 1-25.

Glasberg, B. R., and Moore, B. C. J. (1990). "Derivation of auditory filter shapes from notched-noise data," Hear. Res. 47, 103-138.

Gnansia, D., Jourdes, V., and Lorenzi, C. (2008). "Effect of masker modulation depth on speech masking release," Hear. Res. 239, 60-68.

Grant, K. W. (1987). "Frequency modulation detection by normally hearing and profoundly hearing-impaired listeners," J. Speech Hear. Res. 30, 558563.

Hall, J. W., Tyler, R. S., and Fernandes, M. A. (1984). "Factors influencing the masking level difference in cochlear hearing-impaired and normalhearing listeners," J. Speech Hear. Res. 27, 145-154.

Häusler, R., Colburn, S., and Marr, E. (1983). "Sound localization in subjects with impaired hearing. Spatial-discrimination and interauraldiscrimination tests," Acta Otolaryngol. Suppl. 400, 1-62.

Hawkins, D. B., and Wightman, F. L. (1980). "Interaural time discrimination ability of listeners with sensorineural hearing loss," Audiology 19, 495507.

Hinchcliffe, R. (1992). "King-Kopetzky syndrome: An auditory stress disorder?," J. Audiol. Med. 1, 89-98.

Hopkins, K., and Moore, B. C. J. (2007). "Moderate cochlear hearing loss leads to a reduced ability to use temporal fine structure information," J. Acoust. Soc. Am. 122, 1055-1068.

Hopkins, K., Moore, B. C. J., and Stone, M. A. (2008). "Effects of moderate cochlear hearing loss on the ability to benefit from temporal fine structure information in speech," J. Acoust. Soc. Am. 123, 1140-1153.

Horst, J. W. (1987). "Frequency discrimination of complex signals, frequency selectivity, and speech perception in hearing-impaired subjects," J. Acoust. Soc. Am. 82, 874-885.

Horwitz, A. R., Dubno, J. R., and Ahlstrom, J. B. (2002). "Recognition of low-pass-filtered consonants in noise with normal and impaired highfrequency hearing," J. Acoust. Soc. Am. 111, 409-416.

ISO 389-8 (2004). "Acoustics-Reference zero for the calibration of audiometric equipment—Part 8: Reference equivalent threshold sound pressure levels for pure tones and circumaural earphones," International Organization for Standardization (Geneva).

Joris, P. X., Carney, L. H., Smith, P. H., and Yin, T. C. (1994). "Enhancement of neural synchronization in the anteroventral cochlear nucleus. I. Responses to tones at the characteristic frequency," J. Neurophysiol. 71, 1022-1036.

Kaernbach, C. (1991). "Simple adaptive testing with the weighted up-down method," Percept. Psychophys. 49, 227-229.

King, K., and Stephens, D. (1992). "Auditory and psychological factors in auditory disability with normal hearing," Scandinavian Audiology, 21(2), 109-114.

Kinkel, M., Holube, I., and Kollmeier, B. (1988). "Zusammenhang verschiedener Parameter binauralen Hörens bei Schwerhörigen (Relation between parameters of binaural hearing in hearing impaired subjects)," Fortschritte der Akustik-DAGA 1988 (DPG Kongreß-GmbH, Bad Honnef), pp. 629-632.

Koehnke, J., Culotta, C. P., Hawley, M. L., and Colburn, H. S. (1995). "Effects of reference interaural time and intensity differences on binaural performance in listeners with normal and impaired hearing," Ear Hear. 16, 331-353.

Kunov, H., and Abel, S. M. (1981). "Effects of rise/decay time on the lateralization of interaurally delayed 1-kHz tones," J. Acoust. Soc. Am. 69, 769-773.

Lacher-Fougère, S., and Demany, L. (1998). "Modulation detection by normal and hearing-impaired listeners," Audiology 37, 109-121.

Lacher-Fougère, S., and Demany, L. (2005). "Consequences of cochlear damage for the detection of interaural phase differences," J. Acoust. Soc. Am. 118, 2519-2526.

Laird, N. M., and Ware, J. H. (1982). "Random-effects models for longitu- 
dinal data," Biometrics 38, 963-974.

Levitt, H., and Rabiner, L. R. (1967). "Binaural release from masking for speech and gain in intelligibility," J. Acoust. Soc. Am. 42, 601-608.

Liberman, M. C., and Dodds, L. W. (1984). "Single-neuron labeling and chronic cochlear pathology. III. Stereocilia damage and alterations of threshold tuning curves," Hear. Res. 16, 55-74.

Lorenzi, C., Gilbert, G., Carn, H., Garnier, S., and Moore, B. C. J. (2006). "Speech perception problems of the hearing impaired reflect inability to use temporal fine structure," Proc. Natl. Acad. Sci. U.S.A. 103, 18866 18869

Lorenzi, C., and Moore, B. C. J. (2008). "Role of temporal envelope and fine structure cues in speech perception: A review," in Auditory Signal Processing in Hearing-Impaired Listeners, First International Symposium on Auditory and Audiological Research (ISAAR 2007), edited by T. Dau, J. M. Buchholz, J. M. Harte, and T. U. Christiansen (Centertryk, Denmark). Middelweerd, M. J., Festen, J. M., and Plomp, R. (1990). "Difficulties with speech intelligibility in noise in spite of a normal pure-tone audiogram," Audiology 29, 1-7.

Miller, R. L., Schilling, J. R., Franck, K. R., and Young, E. D. (1997) "Effects of acoustic trauma on the representation of the vowel "eh" in cat auditory nerve fibers," J. Acoust. Soc. Am. 101, 3602-3616.

Moore, B. C. J. (1996). "Perceptual consequences of cochlear hearing loss and their implications for the design of hearing aids," Ear Hear. 17, 133 161.

Moore, B. C. J. (2003). An Introduction to the Psychology of Hearing (Academic, San Diego, CA), pp. 197-204.

Moore, B. C. J. (2008). "The role of temporal fine structure processing in pitch perception, masking, and speech perception for normal-hearing and hearing-impaired people," J. Assoc. Res. Otolaryngol. 9, 399-406.

Moore, B. C. J., Glasberg, B. R., and Baer, T. (1997). "A model for the prediction of thresholds, loudness, and partial loudness," J. Audio Eng Soc. 45, 224-240.

Moore, B. C. J., Glasberg, B. R., and Hopkins, K. (2006). "Frequency discrimination of complex tones by hearing-impaired subjects: Evidence for loss of ability to use temporal fine structure," Hear. Res. 222, 16-27.

Moore, B. C. J., Glasberg, B. R., and Peters, R. W. (1985). "Relative dominance of individual partials in determining the pitch of complex tones," $\mathrm{J}$. Acoust. Soc. Am. 77, 1853-1860.

Moore, B. C. J., Peters, R. W., and Glasberg, B. R. (1990). "Auditory filter shapes at low center frequencies," J. Acoust. Soc. Am. 88, 132-140.

Moore, B. C. J., and Sek, A. (1996). "Detection of frequency modulation at low modulation rates: Evidence for a mechanism based on phase locking," J. Acoust. Soc. Am. 100, 2320-2331.

Moore, B. C. J., and Skrodzka, E. (2002). "Detection of frequency modulation by hearing-impaired listeners: Effects of carrier frequency, modulation rate, and added amplitude modulation," J. Acoust. Soc. Am. 111, 327-335.

Moore, B. C. J., Vickers, D. A., Plack, C. J., and Oxenham, A. J. (1999). "Inter-relationship between different psychoacoustic measures assumed to be related to the cochlear active mechanism," J. Acoust. Soc. Am. 106, 2761-2778.

Narula, A. A., and Mason, S. M. (1988). "Selective dysacusis-A preliminary report," J. R. Soc. Med. 81, 338-340.

Nie, K., Stickney, G., and Zeng, F.-G. (2005). "Encoding frequency modulation to improve cochlear implant performance in noise," IEEE Trans. Biomed. Eng. 52, 64-73.

Noordhoek, I. M., Houtgast, T., and Festen, J. M. (2001). "Relations between intelligibility of narrow-band speech and auditory functions, both in the 1-kHz frequency region," J. Acoust. Soc. Am. 109, 1197-1212.

Oxenham, A. J., and Shera, C. A. (2003). "Estimates of human cochlear tuning at low levels using forward and simultaneous masking," J. Assoc. Res. Otolaryngol. 4, 541-554.

Patterson, R. D., and Moore, B. C. J. (1986). "Auditory filters and excitation patterns as representations of frequency resolution," in Frequency Selectivity in Hearing, edited by B. C. J. Moore (Academic, London).

Patterson, R. D., and Nimmo-Smith, I. (1980). "Off-frequency listening and auditory-filter asymmetry,” J. Acoust. Soc. Am. 67, 229-245.

Patterson, R. D., Nimmo-Smith, I., Weber, D. L., and Milroy, R. (1982). "The deterioration of hearing with age: Frequency selectivity, the critical ratio, the audiogram, and speech threshold," J. Acoust. Soc. Am. 72, 1788-1803.

Peterson, G., and Barney, H. (1952). "Control methods used in a study of the vowels," J. Acoust. Soc. Am. 24, 175-184.

Pichora-Fuller, M. K., and Schneider, B. A. (1992). "The effect of interaural delay of the masker on masking-level differences in young and old adults," J. Acoust. Soc. Am. 91, 2129-2135.

Pinheiro, J., and Bates, D. (2000). Mixed-Effects Models in S and S-PLUS (Springer-Verlag, New York).

Plomp, R. (1978). "Auditory handicap of hearing impairment and the limited benefit of hearing aids," J. Acoust. Soc. Am. 63, 533-549.

Qin, M. K., and Oxenham, A. J. (2003). "Effects of simulated cochlearimplant processing on speech reception in fluctuating maskers," J. Acoust. Soc. Am. 114, 446-454.

Rhode, W. S., Geisler, C. D., and Kennedy, D. T. (1978). “Auditory nerve fiber response to wide-band noise and tone combinations," J. Neurophysiol. 41, 692-704

Rosen, S. (1987). "Phase and the hearing-impaired," in The Psychophysics of Speech Perception, edited by M. E. H. Schouten (Springer-Verlag, New York).

Rosen, S., Baker, R. J., and Darling, A. (1998). "Auditory filter nonlinearity at $2 \mathrm{kHz}$ in normal hearing listeners," J. Acoust. Soc. Am. 103, 25392550 .

Ross, B., Fujioka, T., Tremblay, K. L., and Picton, T. W. (2007). "Aging in binaural hearing begins in mid-life: Evidence from cortical auditoryevoked responses to changes in interaural phase," J. Neurosci. 27, 1117211178.

Ruggero, M. A. (1992). "Physiology and coding of sound in the auditory nerve," in The Mammalian Auditory Pathway: Neurophysiology, edited by A. N. Popper and R. R. Fay (Springer-Verlag, New York), pp. 34-93.

Saberi, K. (1995). "Some considerations on the use of adaptive methods for estimating interaural-delay thresholds," J. Acoust. Soc. Am. 98, 18031806.

Santurette, S., and Dau, T. (2007). "Binaural pitch perception in normalhearing and hearing-impaired listeners," Hear. Res. 223, 29-47.

Saunders, G. H., and Haggard, M. P. (1989). "The clinical assessment of obscure auditory dysfunction-1. Auditory and psychological factors," Ear Hear. 10, 200-208.

Saunders, G. H., and Haggard, M. P. (1992). "The clinical assessment of "obscure auditory dysfunction" (OAD) 2. Case control analysis of determining factors," Ear Hear. 13, 241-254.

Schneider, B. A., Daneman, M., and Pichora-Fuller, M. K. (2002). "Listening in aging adults: From discourse comprehension to psychoacoustics," Can. J. Exp. Psychol. 56, 139-152.

Schubert, E. D., and Schultz, M. C. (1962). "Some aspects of binaural signal selection," J. Acoust. Soc. Am. 34, 844-849.

Schuknecht, H. F., and Woellner, R. C. (1953). "Hearing losses following partial section of the cochlear nerve," Laryngoscope 63, 441-465.

Shamma, S. (2001). "On the role of space and time in auditory processing," Trends Cogn. Sci. 5, 340-348.

Shannon, R. V., Zeng, F. G., Kamath, V., Wygonski, J., and Ekelid, M. (1995). "Speech recognition with primarily temporal cues," Science 270, 303-304.

Smoski, W. J., and Trahiotis, C. (1986). "Discrimination of interaural temporal disparities by normal-hearing listeners and listeners with highfrequency sensorineural hearing loss," J. Acoust. Soc. Am. 79, 1541-1547.

Staffel, J. G., Hall, J. W., Grose, J. H., and Pillsbury, H. C. (1990). "NoSo and $\operatorname{NoS} \pi$ detection as a function of masker bandwidth in normal-hearing and cochlear-impaired listeners," J. Acoust. Soc. Am. 87, 1720-1727.

Stern, M., and Trahiotis, C. (1995). "Models of binaural interaction," in Hearing, edited by B. C. J. Moore (Academic, San Diego, CA).

Strouse, A., Ashmead, D. H., Ohde, R. N., and Grantham, D. W. (1998). "Temporal processing in the aging auditory system," J. Acoust. Soc. Am. 104, 2385-2399.

Terhardt, E. (1974). "Pitch, consonance, and harmony," J. Acoust. Soc. Am. 55, 1061-1069.

Turner, C. W. (1987). "Effects of noise and hearing loss upon frequency discrimination," Audiology 26, 133-140.

Turner, C. W., and Nelson, D. A. (1982). "Frequency discrimination in regions of normal and impaired sensitivity," J. Speech Hear. Res. 25, 34-41.

Tyler, R. S., Wood, E. J., and Fernandes, M. (1983). "Frequency resolution and discrimination of constant and dynamic tones in normal and hearingimpaired listeners," J. Acoust. Soc. Am. 74, 1190-1199.

van Schijndel, N. H., Houtgast, T., and Festen, J. M. (2001). "Effects of degradation of intensity, time, or frequency content on speech intelligibility for normal-hearing and hearing-impaired listeners," J. Acoust. Soc. Am. 110, 529-542.

Wagener, K., Josvassen, J. L., and Ardenkjaer, R. (2003). "Design, optimi- 
zation and evaluation of a Danish sentence test in noise," Int. J. Audiol. 42, 10-17.

Wang, J., Powers, N. L., Hofstetter, P., Trautwein, P., Ding, D., and Salvi, R. (1997). "Effects of selective inner hair cell loss on auditory nerve fiber threshold, tuning and spontaneous and driven discharge rate," Hear. Res. 107, 67-82.

Wightman, F. L., and Kistler, D. J. (1992). "The dominant role of lowfrequency interaural time differences in sound localization," J. Acoust. Soc. Am. 91, 1648-1661.

Woolf, N. K., Ryan, A. F., and Bone, R. C. (1981). "Neural phase-locking properties in the absence of cochlear outer hair-cells," Hear. Res. 4, 335346.

Zeng, F.-G., Nie, K., Liu, S., Stickney, G., Rio, E. D., Kong, Y.-Y., and Chen, H. (2004). "On the dichotomy in auditory perception between temporal envelope and fine structure cues," J. Acoust. Soc. Am. 116, 13511354
Zeng, F.-G., Nie, K., Stickney, G. S., Kong, Y.-Y., Vongphoe, M., Bhargave, A., Wei, C., and Cao, K. (2005). "Speech recognition with amplitude and frequency modulations,” Proc. Natl. Acad. Sci. U.S.A. 102, 2293-2298.

Zhao, F., and Stephens, D. (2000). "Subcategories of patients with KingKopetzky syndrome," Br. J. Audiol. 34, 241-256.

Zhao, F., and Stephens, D. (2006). "Distortion product otoacoustic emissions in patients with King-Kopetzky syndrome," Int. J. Audiol. 45, 3439.

Zurek, P. M. (1993). "A note on onset effects in binaural hearing," J. Acoust. Soc. Am. 93, 1200-1201.

Zurek, P. M., and Formby, C. (1981). "Frequency-discrimination ability of hearing-impaired listeners," J. Speech Hear. Res. 24, 108-112.

Zwicker, E. (1956). "Die elementaren Grundlagen zur Bestimmung der Informationskapazität des Gehörs (The elementary foundations for determining the information capacity of the auditory system)," Acustica 6, $365-381$. 